Opfer kaum rechtfertigen. ${ }^{346}$ Deutlich wird hier erneut das tief sitzende Misstrauen, das konstituierend war für das Deutschlandbild in den drei untersuchten konservativen Zeitungen.

\title{
2.3 Die Implementierung Des Friedensvertrags: Krisen und Konflikte
}

Da der Vertrag von Versailles das zentrale Element der deutsch-britischen Beziehungen und der wichtigste Baustein der europäischen Nachkriegsordnung war, dominierte die Diskussion über seine Umsetzung und seine Auswirkungen die Berichterstattung in der britischen Presse in den folgenden Jahren weiter fast zwangsläufig. Die Debatte wurde auf der einen Seite genährt durch die anhaltende vehemente Ablehnung des Vertrags in Deutschland, die sich in der Berliner Außen- wie der Innenpolitik niederschlug. Auf der anderen Seite gewann sie durch die Interessengegensätze, die sich schon während der Friedenskonferenz zwischen der britischen und der französischen Delegation aufgetan hatten und die in den frühen 1920er Jahren immer deutlicher hervortraten, zunehmend an Schärfe. ${ }^{347}$ Bevor der erstgenannte Aspekt einer detaillierten Betrachtung unterzogen wird, folgen zunächst einige Bemerkungen zu den wachsenden Spannungen zwischen London und Paris. Hauptursache für diese war die unterschiedliche Interpretation der Rolle Deutschlands in den drei Problemkreisen, die die Politik der beiden Siegermächte determinierten. Das waren erstens die Lösung der Finanzprobleme, zweitens der wirtschaftliche Wiederaufbau und drittens die politisch-militärische Sicherheit. 348

Frankreich blieb beherrscht von der Furcht vor seinem östlichen Nachbarn und räumte der Sicherheitsproblematik den Vorrang ein. Das bedeutete, dass Deutschland dauerhaft daran gehindert werden musste, erneut die wirtschaftliche Vormacht und damit die militärische Dominanz in Europa zu erringen. ${ }^{349}$

346 Nach Meinung von Krumeich waren die psychologischen Spätfolgen des Kriegs ausschlaggebend für das Scheitern einer Aussöhnung. „Angesichts der massiven und fortbestehenden Traumatisierung des Weltkriegs, die noch in den konkreten Ausformulierungen des Versailler Vertrags stets spürbar blieb, war der verzweifelt ernsthafte Versuch eines großen Friedens und der Anspruch und der Ehrgeiz seiner Verfasser, durch den einen Akt der Rechtssetzung eine Weltordnung und einen festen gerechten und dauerhaften Frieden zu schaffen, auf tragische Weise unrealisierbar." KRUMEICH, Krieg, S. 64.

347 Die schleichende Entfremdung der beiden Entente-Partner ist Gegenstand zahlreicher Untersuchungen gewesen. Vgl. erst jüngst LOwE, Great Powers sowie BELL, France and Britain. Der folgende kurze Abriss beschränkt sich auf die für das Thema relevanten Aspekte.

348 Vgl. WirsCHING, Großbritanniens Europapolitik, S. 211.

349 Ebd., S. 212. Neuralgischer Punkt war Frankreichs Abhängigkeit von ausländischer Kohle. Der französische Bedarf konnte entweder durch forcierte Reparationsleistungen aus Deutschland gedeckt werden, wofür die Zustimmung Englands nötig war, oder direkt aus den Überschüssen der englischen Kohleförderung. In jedem Fall blieb die französische schwerindustrielle Position - Grundlage jeglicher langfristiger Großmachtstellung - politisch von England abhängig. 
Dahinter stand die in der französischen Öffentlichkeit und der politischen Führungsschicht verbreitete Überzeugung, dass die Deutschen zu Veränderungen prinzipiell nicht fähig waren und nach Revanche strebten. ${ }^{350}$ Hinzu kam, dass die ursprünglich vorgesehenen Garantieverträge mit Großbritannien und den USA nach der Ablehnung des Versailler Vertrags durch den amerikanischen Senat scheiterten, sich also die Sicherheitsfrage für Frankreich bereits ab 1920 erneut mit einer gewissen Dringlichkeit stellte. ${ }^{351}$ Dies war das entscheidende Motiv, das der französischen Exekutionspolitik sowie den Allianzen mit den neu gegründeten Staaten Osteuropas zu Grunde lag.

England als bedeutende Industrie- und Exportnation musste hingegen auf Grund der eigenen wirtschaftlichen Probleme in erster Linie an einer dauerhaften Friedensordnung und einer Wiederherstellung des Welthandels interessiert sein, wofür die Existenz Deutschlands als funktionierende Volkswirtschaft und damit als aufnahmefähiger Absatzmarkt erforderlich war. Nur in einem solchen globalen System konnte Großbritannien seine traditionelle Rolle als Finanz-, Handels- und Dienstleistungszentrum wieder aufnehmen und seine Weltmachtposition sichern. In der englischen Politik wuchs deshalb die Bereitschaft, eine Rekonstruktionspolitik zu verfolgen, die einen Wiederaufbau Deutschlands einschloss und eine dauerhafte Schädigung seines wirtschaftlichen Potenzials oder seiner Kreditwürdigkeit zu verhindern suchte. ${ }^{352}$ Ein stabiles Deutschland unter demokratischer Führung war außerdem der beste Schutz gegen eine Verbreitung des Bolschewismus in Richtung Westen, die viele britische Konservative nach wie vor fürchteten. ${ }^{353}$

Dass Frankreich und Großbritannien ihre vitalen Interessen in Bezug auf Deutschland so unterschiedlich definierten, hatte eine Belastungsprobe der Entente Cordiale zur Folge, auch wenn es auf beiden Seiten an gutem Willen zur Zusammenarbeit im Interesse Europas nicht mangelte. ${ }^{354}$ Tatsächlich waren die Friedensentwürfe der beiden Siegermächte aber inkompatibel. Da keine von beiden sich stark genug erwies, ihre Interessen durchzusetzen, verlegten sie sich darauf, die Politik des anderen zu behindern, so dass sich zwischen 1920 und 1923 allmählich eine völlige Blockade einstellte. ${ }^{355}$

Erschwerend kam hinzu, dass sich in Großbritannien eine Wahrnehmungsverschiebung vollzog. Aus britischer Perspektive stellte Deutschland keine unmittelbare Gefahr mehr dar, nachdem es gemäß dem Waffenstillstand und dem Friedensvertrag sowohl seine Schlacht- als auch seine Handelsflotte hatte aus-

350 BELL, France and Britain, S. 118.

351 BARIÉTY, Sicherheitsfrage, S. 329.

352 Wirsching, Großbritanniens Europapolitik, S.211. Ebenso KLEINE-AhlbrandT, Burden, S. 55.

353 DOERR, Foreign Policy, S. 32.

354 BENNETT, British Foreign Policy, S. 14.

355 BeLL, France and Britain, S.134f. Ebenso WirschING, Großbritanniens Europapolitik, S. 213. 
liefern müssen, seine Kolonien verloren hatte, keine Luftwaffe und nur noch ein Heer von 100000 Mann besitzen durfte, sowie der Liquidation seiner Auslandsvermögen und der Beseitigung seiner Zollhoheit hatte zustimmen müssen. Stattdessen wuchs jetzt, insbesondere in der politischen Führungsschicht Englands, die Furcht vor einer französischen Hegemonie.

Germany could no longer stand in the way of French ambitions in Europe. AustriaHungary had dissolved into a number of smaller states which were rapidly pulled into the French orbit. Russia was in chaos. France, with her large conscript army, was excellently placed to dominate European affairs. [...] Consequently, Britain was keenly interested in resisting the spread of French power and influence. ${ }^{356}$

Ängste dieser Art schienen sich durch französische Alleingänge wie die Besetzung von Frankfurt, Darmstadt und Hanau als Reaktion auf die Verlegung von Reichswehreinheiten in die entmilitarisierte Zone entlang des Rheins im April $1920 \mathrm{zu}$ bestätigen. ${ }^{357}$ Die Verärgerung der britischen Regierung über diese Aktion war so groß, dass man sich zu einem öffentlichen Protest gegen die militaristische Politik Frankreichs genötigt sah. ${ }^{358}$ Wie Austen Chamberlain hielten viele Mitglieder des Kabinetts den Kurs der französischen Führung für gefährlich, da er zusätzliche Spannungen erzeugte und auf deutscher Seite erst recht Widerstand provozierte. ${ }^{359}$

Der Vorgang ist charakteristisch für die gegensätzlichen Konzeptionen der beiden Siegermächte. Während Premierminister Lloyd George eine freiwillige Erfüllung des Versailler Vertrags auf der Grundlage eines Konsenses zwischen den Alliierten und Deutschland anstrebte, der mithilfe der Konferenzdiplomatie herbeigeführt werden sollte, setzten französische Regierungschefs auf eine buchstabengetreue Einhaltung und im Verzugsfall auf die Drohung mit Sanktionen. ${ }^{360}$ Im Fokus beider Seiten stand dabei das Problem der Re-

356 BenNeTT, British Foreign Policy, S. 13.

357 Die deutsche Regierung begründete die temporäre Entsendung von Truppen mit der Notwendigkeit, die Rote Armee, die sich im Gefolge des Kapp-Lüttwitz-Putsches im Ruhrgebiet gegründet hatte, aufzulösen. Die britische Regierung hielt diesen Schritt nicht für eine so gravierende Verletzung des Friedensvertrags, als dass sie eine militärische Antwort der Alliierten erforderte. Vgl. BELL, France and Britain, S. 135.

358 Das Kabinett beauftragte Sir Eyre Crowe und Philip Kerr, durch das News Department des Foreign Office der Presse die britische Haltung zu erklären und zu begründen. Protokoll der Kabinettssitzung, 8. April 1920, PRO, CAB 23/21. Es war einer der wenigen Fälle in dieser Zeit, in denen das Kabinett einen offiziellen Beschluss über die Information der Presse fasste.

359 Chamberlain hielt die öffentliche Distanzierung von Großbritanniens wichtigstem Partner deshalb für angemessen und an der Zeit. „it was time to let them [die Franzosen, der Verf.] know that if they provoked or provoke a row by isolated action, they will be left to settle it by themselves as best as they can. They live in a nightmare terror of Germany, but unless they are careful, they will plant such memories as Germany will never forget, and some day will avenge." Zit. nach PETRIE, Life and Letters, Bd. 1, S. 155.

360 Wirsching, Großbritanniens Europapolitik, S.214. Wie Georges-Henri Soutou und Jacques Bariéty herausgearbeitet haben, war die Politik Frankreichs nicht so unflexibel, 
parationen. Nach der Festsetzung der Gesamtsumme und der Annahme des Zahlungsplans auf der Londoner Konferenz Anfang Mai 1921 entbrannte der Streit um die Legitimität der alliierten Forderungen, ihre Höhe und die Art der Begleichung in vollem Umfang. Frankreich war auf die deutschen Zahlungen und Sachleistungen angewiesen, um seine Kriegsschäden zu beheben, und sah darüber hinaus in den Reparationen ein Instrument, um Deutschland auf Dauer zu schwächen. ${ }^{361}$ In Großbritannien herrschte dagegen die Furcht, dass zu hohe Reparationslasten die wirtschaftliche Erholung Deutschlands und damit ganz Europas zum eigenen Schaden verlangsamen könnten. ${ }^{362}$

Die offenen Differenzen waren Wasser auf die Mühlen der Vertragskritiker, die entsprechend nicht an Polemik sparten. Seit John Maynard Keynes mit den finanziellen Bestimmungen in seinem Buch The Economic Consequences of the Peace abgerechnet hatte, das im Dezember 1919 erschienen war, standen diese ohnehin unter Beschuss. ${ }^{363}$ Hinzu kam bei Labournahen und liberalen Publizisten nun der Eindruck, dass Frankreich die Strategie verfolgte, unter dem Deckmantel des Friedensvertrags ohne Absprache offensichtlich unerfüllbare Forderungen aufzustellen, um dann wiederum einseitig - zu erklären, dass Deutschland seinen Verpflichtungen nicht nachkam. ${ }^{364}$ Die konservative Presse, allen voran die Zeitungen Northcliffes, übernahm hingegen den französischen Standpunkt und pochte Punkt für Punkt auf eine Erfüllung des Vertrags, obwohl auch

wie sie auf den ersten Blick schien. Unter Millerand entstand z.B. ein Vorschlag, die Reparationsfrage durch eine allgemeine ökonomische Verzahnung der Wirtschaft Frankreichs und Deutschlands zu lösen, der so genannte „Seydoux-Plan“ aus dem Frühjahr 1920. Dieser war jedoch zu diesem Zeitpunkt weder für Berlin noch für London annehmbar. Vgl. SouTOu, System von Versailles, S.79f. Insgesamt schwankte die französische Deutschlandpolitik in den frühen 20er Jahren zwischen Drohungen mit einer Macht- und Sanktionspolitik und Versuchen, einen Kompromiss zu finden. Vgl. BARIÉTY, Französische Politik, S. 17.

361 Doerr, Foreign Policy, S. 57-59.

362 Ebd.

363 Der Einfluss von Keynes auf die Debatte in der englischen Presse ist schwer einzuschätzen. Sein Rückzug aus der britischen Delegation in Paris im Juni 1919 war den hier untersuchten Zeitungen keine Meldung wert. Nach dem Erscheinen seines Buches gab ihm Scott dann im Manchester Guardian in den Jahren 1920 bis 1923 immer wieder die Gelegenheit, zum Thema Reparationen und den Folgeproblemen Stellung zu nehmen, und auch die Times beachtete seine Analysen. Eine fundierte Studie über Keynes' Anteil am öffentlichen Diskurs, der die Legitimität des Versailler Vertrag nach und nach untergrub, steht aber noch aus und wird auch von Peter Matthias in seiner Abhandlung über Keynes und die britische Deutschlandpolitik nur am Rande thematisiert.

364 Brailsford erhob den Vorwurf, dass Paris seine militärische Überlegenheit und seine damit verbundene Vormacht als „Mistress of Europe“ rücksichtslos ausnutze. THE DAILY HERALD, 3. Mai 1921, LA „Back To Napoleon“. Der Guardian forderte sogar, Frankreich die Zusammenarbeit aufzukündigen, falls es an dieser Art von Außenpolitik festhielt. THE MANCHESTER Guardian, 3. Mai 1921, LA „How Much Farther“. 
hier einige Argumente der Kritik von Keynes eine gewisse Resonanz fanden. ${ }^{365}$

Der deutschen Seite bot die Uneinigkeit der Siegermächte die Möglichkeit, beide gegeneinander auszuspielen. Unter Verweis auf die eigenen wirtschaftlichen und fiskalischen Probleme versuchten die Regierungen in Berlin zu zeigen, dass die Reparationsforderungen nicht nur übertrieben hoch und deshalb unbezahlbar, sondern auch ungerecht waren. Dahinter stand die Absicht, die Legitimität des gesamten Vertragswerks zu erschüttern. ${ }^{366}$ Die deutschen Proteste erstreckten sich auch auf andere Bereiche, etwa auf die Prozesse gegen deutsche Kriegsverbrecher oder die Volksabstimmung in Oberschlesien.

Die Reaktionen darauf waren in London gespalten: Die breite Ablehnung des Versailler Vertrags quer durch alle Parteien und gesellschaftlichen Gruppen stieß auf wenig Verständnis. Außerdem vermisste man ernsthafte Anstrengungen zur Erfüllung der alliierten Auflagen und fürchtete, dass die Weimarer Politiker Konzessionen der Siegermächte zum Anlass nehmen würden, um weitere Zugeständnisse zu fordern. ${ }^{367}$ Andererseits wuchs das Verständnis für die Beschwerden aus Berlin, zumal offenkundig war, in welch schwierige Lage die dortigen Regierungen geraten konnten, wenn sie eine Politik der Erfüllung verfolgten. Der oberste britische Repräsentant in Deutschland, Lord Kilmarnock, hatte deshalb schon Ende Januar 1920 empfohlen, Entgegenkommen überall dort zu zeigen, wo dies möglich war, gerade um die Weimarer Republik zu stabilisieren; eine Überlegung, der die Führungsspitze des Foreign Office im Grundsatz zustimmte. ${ }^{368}$ Die Befürworter eines solchen Kurses sahen in Entlastungen etwa bei der Reparationsregelung, teils auch in anderen Fragen, ein Mittel, um verständigungsbereiten Kräften in Berlin unter die Arme zu greifen und so deren „Erfüllungspolitik“ zu belohnen. Doch war strittig, wie weit London durch Zugeständnisse in einzelnen Punkten die Konsolidierung der politischen Mitte in Deutschland unterstützen konnte und sollte. ${ }^{369}$

365 Die Times, die Daily Mail aber auch der Daily Telegraph bestanden darauf, dass Deutschland den Friedensvertrag und die alliierten Reparationsforderungen erfüllen müsse, bevor über mögliche Änderungen verhandelt werden könnte. In diesem Sinne äußerten sich alle Kommentatoren der drei Blätter z.B. zum schon erwähnten Londoner Ultimatum. Vgl. The Times, 3. Mai 1921, LA „The One Issue“; The Daily Mail, 3. Mai 1921, LA „Settle Up - And Be Done With It“; The Daily Telegraph, 2. Mai 1921, LA „Conference Problems". Das Buch von Keynes hatte Steed in der Times rezensiert und darin dessen Kritik an der vorläufigen Regelung der Reparationsfrage im Versailler Vertrag als berechtigt bezeichnet. Die übrigen Vorwürfe insbesondere gegen die „Großen Drei“ Lloyd George, Wilson und Clemenceau konnte er jedoch nicht nachvollziehen. THE TIMES, 5. Januar 1920, „A Critic Of The Peace - Comfort For Germany“.

366 LOwe, Great Powers, S. 247-249.

367 RECKER, Neuordnung, S. 103.

368 DBFP, I, 9, Nr.23, S.28, Kilmarnock an Curzon, 31.Januar 1920. Die Führungsspitze des Foreign Office hielt diese Überlegung für sehr wichtig, so die Notiz von Curzon und Hardinge.

369 ReCKER, Neuordnung, S. 103. 
Exemplarisch für den Verlauf dieser Diskussion, die nicht nur in der Politik, sondern mit den gleichen Argumenten auch in der Presse geführt wurde, ist die Korrespondenz zwischen dem Chefredakteur der Times, Wickham Steed, und dem ehemaligen Berliner Korrespondenten George Saunders, der zwischenzeitlich als Deutschlandexperte für das Foreign Office gearbeitet hatte, anzusehen. ${ }^{370}$ Saunders reiste im März 1920 in die deutsche Hauptstadt, um im Auftrag der renommierten Zeitung Reportagen über die aktuelle politische und wirtschaftliche Situation zu verfassen. Dort angekommen geriet er mitten in den Kapp-Lüttwitz-Putsch. Für den reaktionären Umsturzversuch war seiner Ansicht nach der Versailler Vertrag mittelbar und die französische Auslegung desselben unmittelbar verantwortlich. ${ }^{371}$

The French policy is to let Germany stew in her own juice, meanwhile squeezing out for France what drops of that juice can be extracted. It is, in my opinion, a suicidal policy for France and for all of us. It is absurd to expect Germany to preserve order here and throughout the Reich with 100000 men. We must get on terms of business and realise what is necessary to make life liveable here, before we proceed to legitimate exactions. ${ }^{372}$

Steed hingegen war der Auffassung, dass die Allianz mit Frankreich auf keinen Fall in Frage gestellt und den Deutschen nicht der Eindruck vermittelt werden durfte, "that we are ready to throw over the French for beautiful Teutonic eyes“. Nicht die französische Außenpolitik war seiner Meinung nach das eigentliche Problem, sondern die Deutschen.

The root of the trouble is that the Germans do not yet seem to know that they have been beaten and still less that they themselves are responsible for the beating they got. That they will only understand when by hook or by crook they have been disarmed. The Allies should then fix, I care not how moderate a sum, the amount of reparations which must be paid. ${ }^{373}$

Auf deutsche Forderungen konnte erst eingegangen werden, wenn die Deutschen einen Beweis für ihre Vertrauenswürdigkeit geliefert hatten, so Steed. ${ }^{374}$

370 Im Political Intelligence Department. Von 1897 bis 1908 hatte Saunders für die Times aus Deutschland berichtet und zu den anti-deutschen Hardlinern gehört, die die Welt über den wahren Charakter der wilhelminischen Politik und ihrer militaristischen Tendenzen aufklären wollte. WILSON (Hrsg.), Saunders on Germany, Einleitung S.1f. In dieser Sammlung ist auch der wesentliche Teil des Briefwechsels mit Steed abgedruckt.

371 U.a. teilte auch Premierminister Lloyd George diese Ansicht. Nachdem dieser die Nachricht von einer reaktionären Revolution in Deutschland erhalten hatte, erklärte er gegenüber Riddell: „the French had helped to bring about the revolution by making and enforcing demands which had made the position of the German Government impossible." RIDDELL, Diaries, Eintrag vom 20./21. März 1920.

372 Saunders an Steed, 19. März 1920, Steed Papers, Correspondence with George Saunders, TNL Archive, HWS/1.

373 Ebd.

374 Ebd. 
Die Schwierigkeiten bei der Umsetzung des Friedensvertrags, die aus der komplizierten Gemengelage gegensätzlicher Interessen Großbritanniens und Frankreichs sowie der zunehmend unterschiedlichen Wahrnehmung Deutschlands in diesen beiden Ländern resultierten, führten dazu, dass in weiten Teilen der britischen Öffentlichkeit die Begeisterung für eine Bestrafung des ehemaligen Kriegsgegners nach 1918 in nur wenigen Jahren deutlich nachließ. ${ }^{375}$ Viele Briten hatten den Respekt für das deutsche Volk und seine Leistungen in der Vergangenheit trotz der Gräuelpropaganda nicht verloren und wünschten eine Normalisierung der Beziehungen. ${ }^{376}$

Die anhaltenden Spannungen zwischen Frankreich und Deutschland entluden sich im Januar 1923 schließlich in der Ruhrbesetzung, die als Katalysator für die bereits begonnene Veränderung des britischen Urteils über Deutschland wirkte. Im Folgenden wird nachgezeichnet, wie sich dieser Wandel in der englischen Presse niederschlug und inwiefern hier Zusammenhänge mit dem Deutschlandbild in der Politik bestanden. Zwei weitere wichtiger Aspekt sind, mit welchen Mitteln die Reichsregierung über ihre Presseabteilung das Bild Deutschlands im Ausland während des Ruhrkampfes zu beeinflussen suchte und ob sie damit Erfolg hatte.

\subsubsection{Die Rubrbesetzung: Deutschland als Opfer französischer Aggression}

Der Einmarsch französischer und belgischer Truppen in das Ruhrgebiet kam nicht überraschend, sondern hatte sich lange vorher angekündigt. Bereits am 24. April 1922 hatte der französische Ministerpräsident Poincaré in seiner Rede in Bar-le-Duc mit einem Alleingang seines Landes bei der Verhängung von Sanktionen gegen ein vertragsbrüchiges und zahlungsunwilliges Deutschland gedroht. ${ }^{377}$ Die britische Regierung vertrat hingegen den Standpunkt, dass gemäß dem Versailler Vertrag Sanktionen nur von den Siegermächten gemeinsam verhängt werden durften, ganz abgesehen davon, dass hier ernsthafte Zweifel bestanden, ob Deutschland überhaupt in der Lage war, seinen Reparationsverpflichtungen nachkommen zu können. Der Dissens verschärfte sich bei den

375 RobBins, Present and Past, S. 30.

376 Kleine-AhlbrandT, Burden, S. 57.

377 Für die unnachgiebige Haltung von Poincaré waren mehrere Gründe maßgeblich: angefangen vom Abschluss des Rapallo-Vertrags zwischen Deutschland und Russland über die Herabsetzung der Reparationen bei der Expertenkonferenz im Juni in Paris und die andauernde Finanz- und Währungskrise des Reiches bis hin zur Balfour-Note über die interalliierten Schulden. Als Folge all dessen drohte Frankreich im Verlauf des Jahres 1922 der Sieg im Weltkrieg doch noch zu entgleiten. SouTOU, System von Versailles, S. 82 und neuerdings SOUTOU, Vom Rhein zur Ruhr, S. 63-67. Über die Bedeutung der interalliierten Schulden siehe insbesondere ARTAUD, Hintergründe, S. 241-259. Zu Ursachen und Verlauf der Ruhrkrise allgemein sowie der britischen Rolle vgl. auch WiLliamson, Ruhr Crisis, S. 70-91 sowie SCHWABE, Großbritannien, S. 53-87. 
Beratungen der Hauptreparationsgläubiger über einen Antrag Deutschlands auf ein Moratorium auf der Londoner Konferenz im August 1922. Poincaré stellte das Junktim auf, dass es ohne „produktive Pfänder“ keinen Zahlungsaufschub geben könne, während Lloyd George unterstützt von seinem Kabinett entschlossen war, den Franzosen keine Zugeständnisse zu machen. ${ }^{378}$ Nachdem der Regierungswechsel in London Ende Oktober keine Veränderung der Rechtsauffassung Großbritanniens zur Folge hatte und Paris ebenfalls auf seinem Standpunkt beharrte, war klar, dass eine Kraftprobe zwischen den eigentlichen Kontrahenten Frankreich und Deutschland nicht mehr aufzuhalten war.

Die Krise brachte die britische Führung in eine prekäre Position. Das Kabinett sah sich vor die Wahl gestellt, entweder einen offenen Bruch mit Frankreich zu riskieren, was aller Voraussicht nach dem Versailler Vertrag die Grundlage entzogen und Europa ins Chaos gestürzt hätte, oder einzulenken und sich Paris anzuschließen. ${ }^{379}$ Um diesem Konflikt zu entkommen, entschied man sich für eine Politik der Inaktivität, die mit dem Begriff der „wohlwollenden Neutralität“ versehen wurde. ${ }^{380}$ Auf diese Weise hoffte man, die Entente Cordiale und damit den Einfluss auf Frankreich retten zu können und die Deutschen nicht zusätzlich zum Widerstand anzuspornen, womit man bei einer eindeutigen Parteinahme für Berlin rechnete. ${ }^{381}$

Da sowohl die französische als auch die deutsche Regierung von London Unterstützung und einen Beitrag zur Lösung des Konflikts erwarteten, versuchten beide von Beginn der Ruhrbesetzung an, die öffentliche Meinung in Großbritannien zu ihren Gunsten zu beeinflussen. Die Propagandaschlacht, die sich Berlin und Paris lieferten, war ein wichtiger Teil des Kampfes um die Ruhr. ${ }^{382}$ Im Mittelpunkt der pressepolitischen Aktivitäten standen dabei insbesondere die britischen Auslandskorrespondenten in Deutschland. Die französischen Besatzungsbehörden im Ruhrgebiet und die Presseabteilung der Reichsregierung bemühten sich, diese mit Informationen über das Geschehen und entsprechenden Interpretationen desselben $\mathrm{zu}$ versorgen. Beide Stellen bauten zu diesem Zweck eigene Nachrichtenorganisationen im besetzten Gebiet auf.

378 ARTAUd, Hintergründe, S. 249.

379 Premierminister Bonar Law sagte wörtlich zu C.P. Scott: „I may have to choose between two evils - between a break with France, which would mean chaos in Europe, or concessions to France, which would also involve great mischief." Scott Papers, Political Diaries, Eintrag vom 6. Dezember 1922, MGA, Box 134.

380 Urheber dieses Ausdrucks war Miles Lampson, der Leiter des Central Department des Foreign Office. Williamson, Ruhr Crisis, S. 70, Fn. 5.

381 Nicht ohne Grund, denn die deutsche Regierung unter Reichskanzler Cuno setzte in der Tat auf die „englische Karte“ und hoffte auf aktive Unterstützung aus London. Vgl. WEIDENFELD, Englandpolitik, S. $62 \mathrm{f}$.

$382 \mathrm{Zu}$ Organisation und Wirkung der deutschen Propagandaaktivitäten siehe MüLLER, Auswärtige Pressepolitik, Kapitel III. 
Die Presseabteilung entsandte noch vor dem Einmarsch der Franzosen ihre zwei Referenten, Zechlin und Brammer, nach Essen. ${ }^{383}$ Diese veranstalteten zweimal täglich im Hotel Kaiserhof, wo die Berliner Korrespondenten der ausländischen Zeitungen wohnten, Pressekonferenzen. Dabei versuchten sie nach eigenen Angaben, durch möglichst „objektive Tatsachenberichte“ vor allem das Vertrauen der amerikanischen und britischen Journalisten $\mathrm{zu}$ gewinnen, um so ein Fundament zu legen, „auf dem auch andere Nachrichten gegeben werden konnten, z.B. Zahlen über Requisitionen, Mitteilungen über Milchversorgung, Kindernot usw." 384 Auf diese Weise wollten sie den Einfluss der belgischen und französischen Besatzungsarmee konterkarieren, die die Reporter an der Ruhr mit „vorzüglichem, in ihrem Sinne wirkendem Informationsmaterial“ versorgten, wie die Presseabteilung besorgt beobachtete. ${ }^{385}$ Besonders die Korrespondenten der Daily Mail, Phillips und Cardozo, fielen dadurch auf, dass sie sich überwiegend auf französische Quellen stützten und ihre eigene Regierung wegen deren Passivität scharf angriffen. ${ }^{386}$ Die anderen englischen Journalisten lehnten das französische Vorgehen dagegen ab, wollten aber ausdrücklich nicht als „deutschfreundlich“ angesehen werden. ${ }^{387}$

Im Wettstreit um die Meinungsführerschaft kam es in der Anfangsphase der Ruhrbesetzung trotz räumlicher Trennung fast täglich zum direkten Aufeinandertreffen der Propagandisten. Für die Franzosen hatte Francois Poncet in Düsseldorf einen Pressestab eingerichtet, weil sich dort die Korrespondenten aus Paris einquartiert hatten. Um dessen Verlautbarungen überwachen und gegebenenfalls unmittelbar darauf reagieren zu können, verlegte Zechlin seinen Standort für einige Zeit von Essen nach Düsseldorf. Poncet wiederum schickte seinen Mitarbeiter Bernaud mit demselben Auftrag in den Kaiserhof nach Essen. ${ }^{388}$

383 ZeChlin, Pressechef, S. 41. Über ihre Aktivitäten zwischen Januar und Februar 1923 berichtete Brammer ausführlich in einem mehrseitigen Bericht. „Vertrauliche Aufzeichnung über die Lage im Ruhrgebiet, über deutsche Aufklärungsarbeit und französische Propaganda“, 23. Februar 1923, ADAP, A, 7, 104, S. 230-236. In Köln bemühte sich mit Noebel ein weiterer Mitarbeiter um die ausländischen Journalisten. Außerdem hatte die Presseabteilung in Münster eine Relaisstation eingerichtet, die alle Nachrichten aus dem besetzten Gebiet nach Berlin meldete und die Verbindung zu den Referenten in Essen hielt. MÜLLER, Auswärtige Pressepolitik, S.113.

384 Außerdem leisteten sie den Journalisten praktische Hilfe, etwa bei der Herstellung von Telefonverbindungen ins Ausland oder der Ausstellung von Passierscheinen. Brammer, „Vertrauliche Aufzeichnung“, 23. Februar 1923, ADAP, A, 7, 104, S. 230-236. Hier S. 233f.

385 Vgl. die Auswertung der entsprechenden Akten in MÜLlER, Auswärtige Pressepolitik, S. 115.

386 Aufzeichnung des Englandreferenten der Presseabteilung Müller-Heymer, „Charakteristik der englischen Zeitungen und ihrer in das Ruhrgebiet entsandten Berichterstatter", 25. Januar 1923, Zit. nach ebd. Seine Kollegen stuften Phillips als „French in sympathy“ ein. Nevinson, Last Changes, S. 291.

387 Brammer, „Vertrauliche Aufzeichnung“, 23. Februar 1923, ADAP, A, 7, 104, S. 230-236. Hier S. 234.

388 Ebd., S. 235. Vgl. auch ZeCHLIn, Pressechef, S. 45. 
Das Ergebnis war, dass die Franzosen im Anschluss an die deutschen Pressekonferenzen ebenfalls Pressekonferenzen in eben diesem Hotel abhielten. ${ }^{389} \mathrm{Da}$ auf diese Weise der Darstellung von einer Seite sofort die Gegendarstellung der anderen folgte, war für die Journalisten leicht zu erkennen, dass sie instrumentalisiert werden sollten. Dem Rheinlandkorrespondenten der Times, Gedye, war klar, dass beide Seiten „massenhaft Propaganda“ abluden. ${ }^{390}$ Dabei entbehrten die Veranstaltungen nicht einer gewissen Komik. Laut Gedye entließ der deutsche Pressereferent Brammer die Journalisten mehrfach mit folgender Bemerkung in die Obhut seines französischen Kollegen: „Aber meine Herren, ich sehe meinen feindlichen Kollegen Birnon [sic. Bernaud, der Verf.] in der Halle warten, um ihnen zu erzählen, was ich für ein Lügner bin, und mich dann selbst zu übertreffen zu suchen, also auf Wiedersehen! "391 In seiner Analyse vom Februar bescheinigte Brammer Bernaud zwar Geschicklichkeit im Umgang mit den Journalisten. Die französische Propaganda sei aber bei Briten und Amerikanern alles in allem nicht sehr erfolgreich. ${ }^{392}$

Um den deutschen Propagandaaktivitäten im von ihnen besetzten Gebiet nicht nur entgegen zu wirken, sondern sie gänzlich zu unterbinden, setzte die französische Militärbehörde auch Zwangsmittel ein. Ende März wurde Zechlin verhaftet und nach einer Woche Gewahrsam aus dem Ruhrgebiet ausgewiesen. ${ }^{393}$ Brammer konnte die Pressearbeit zwar fortsetzen, musste aber, um nicht das gleich Schicksal zu erleiden, häufige Ortswechsel vornehmen und andere Vorsichtsmaßnahmen treffen. ${ }^{394}$

Neben der Information der Reporter im Ruhrgebiet versuchte die Presseabteilung, die in Berlin ansässigen ausländischen Journalisten für die deutsche Seite einzunehmen. Schwierig war das bei englischen Korrespondenten, die gegenüber der deutschen Politik skeptisch eingestellt und selbst noch nicht an der Ruhr gewesen waren, um sich mit eigenen Augen ein Bild von den dortigen Verhältnissen zu machen. Ein Besuch vor Ort führte in vielen Fällen zu einer eindeutigen Parteinahme für eine der beiden Seiten - meistens der deut-

389 Was zweimal täglich der Fall war. GEDYE, Revolver-Republik, S. 92.

390 Ebd., S. 91.

391 Ebd., S. 92.

392 Brammer, „Vertrauliche Aufzeichnung“, 23. Februar 1923, ADAP, A, 7, 104, S. 230-236. Hier S.235. Bei den englischen und amerikanischen Korrespondenten firmierte das französische Pressebüro in Düsseldorf unter der Bezeichnung „Giftküche“. THE MANCHESTER GuARDian, 14. März 1923.

393 Die französischen Behörden versuchten, Zechlins Presseaktivitäten in einen Zusammenhang mit einem blutigen Zwischenfall in den Essener Krupp-Werken zu bringen, wo bei einem Zusammenstoß mit französischen Soldaten 13 deutsche Arbeiter ums Leben gekommen waren. Die ausländischen Journalisten setzten sich für Zechlin ein und protestierten bei den Franzosen, weil ihnen durch seine Verhaftung eine Fülle von Informationen entgingen. ZECHLIN, Pressechef, S. 45-47.

394 Müller, Auswärtige Pressepolitik, S. 114.f 
schen. ${ }^{395}$ Nach einer dreitägigen Reise durch das Rheinland und das Ruhrgebiet erklärte beispielsweise der Berliner Korrespondent der antideutsch eingestellten Morning Post gegenüber Gedye:

In Berlin we have put the stories of the state of the Ruhr down to German propaganda, but now that I have seen for myself what is going on, I feel that we have failed to realise the truth because of the rottenness of the German propaganda system - they have presented understatements of the truth in such an unconvincing way that they read as gross exaggerations. ${ }^{396}$

Parallel zu den Aktivitäten der Presseabteilung versuchte die deutsche Botschaft in London, Informationen in der britischen Presse zu lancieren, die der deutschen Sache dienlich waren. ${ }^{397}$ Gemäß der Berliner Propagandastrategie, wonach die Ruhrbesetzung als „Überfall auf eine friedliche Bevölkerung“ mit dem Ziel der „Zerstückelung des Reiches“ und der „Vernichtung seiner Wirtschaft" gebrandmarkt werden sollte, ${ }^{398}$ ging es dabei z.B. um die wirtschaftliche Bedeutung der linksrheinischen Gebiete und ihre Vernetzung mit dem übrigen Reich. Botschafter Sthamer war darauf bedacht, dass diese Form der Pressearbeit nicht als Propaganda zu erkennen war, da sie sich sonst als kontraproduktiv erweisen konnte. Seiner Beobachtung nach hatte die Ruhrbesetzung den Effekt, dass in manchen Kreisen der Bevölkerung ein antifranzösischer Meinungsumschwung eingetreten war, während in anderen die Kriegsstimmung wieder erwachte. Letzteres führte er u.a. auf den „unheilvollen Einfluss“ der Rothermere-Presse zurück. ${ }^{399}$

Die Daily Mail, das Flaggschiff im Konzern dieses Pressebarons, verfolgte während der gesamten Ruhrkrise eine dezidiert profranzösische Berichterstattung. Die Argumentation war die gleiche, die das Massenblatt seit dem Abschluss des Friedensvertrags verfolgt hatte, nämlich dass sich die Deutschen im Grunde nicht geändert und die Siegermächte mit ihren halbherzigen Bemühungen, den Vertrag zu erfüllen, an der Nase herumführten hätten. „The Germans have persistently cheated us in the past. If experience counts for anything, they will do so again“, so der Tenor. ${ }^{400}$ Aus den Leitartikeln der Mail sprach die

395 Andere britische Besucher, ob das Unternehmer, Labour-Politiker, Radikalliberale wie Lady Bonham Carter oder Studenten von der Nottingham University waren, ergriffen ebenfalls fast alle für die deutsche Seite Partei. Vgl. dazu Thomas, Traveller, S.222, Williamson, Ruhr Crisis, S. 80 und D’Abernon, Red Cross, Eintrag vom 19. März 1923, S. $106 f$.

396 Gedye an Deakin, 10. Juli 1923, Correspondence between Ralph Deakin and G.E.R. Gedye, TNL Archive, TT/FN/1/RD/1. Die Morning Post hatte sich ähnlich wie die Daily Mail für eine Beteiligung Großbritanniens an der Ruhrbesetzung ausgesprochen. BENNETT, British Foreign Policy, S. 34.

397 Bericht Sthamers an das AA, 6. März 1923, ADAP, A, 7, 126, S. 305-307. Der Botschafter hatte dazu nach eigenen Angaben zwei englische Journalisten an der Hand, die ,ausgezeichnete Beziehungen zur hiesigen Presse haben und gewillt sind, in der für das britische Publikum geeigneten Weise zu schreiben“.

398 MÜller, Auswärtige Pressepolitik, S. 108.

399 Bericht Sthamers an das AA, 6. März 1923, ADAP, A, 7, 126, S. 305-307. Hier S. 306.

400 The Daily MaIl, 4. Januar 1923, LA „Why Did He Do It?“. 
Zufriedenheit, dass endlich zumindest eine der Siegermächte energisch gegen die Deutschen vorging. Die Linie der Boulevardzeitung gab Rothermere in einem Artikel mit der plakativen Überschrift „Hats Off To France“ persönlich vor. ${ }^{401}$ Darin erinnerte er an die Verwüstungen, die von den Deutschen 1914 bei der Invasion Frankreichs angerichtet worden waren, dass sie dafür bisher nur geringfügige Zahlungen geleistet hatten und das, obwohl Deutschland in vielerlei Hinsicht „das reichste Land in Europa“ war. Seiner Ansicht nach hatten erst die Divergenzen zwischen England und Frankreich die Deutschen zu ihrer Verweigerungshaltung ermuntert. „While our Parliamentarians have paltered with the German problem and have sought to excuse Germany's most wilful default, France has taken the only logical course to make Germany pay“, schloss er. Die Daily Mail druckte darüber hinaus immer wieder Leserbriefe $\mathrm{ab}$, in denen die französische Politik gutgeheißen wurde, und behauptete, dass die Mehrheit der britischen Öffentlichkeit Frankreich unterstütze. ${ }^{402}$ Den Widerstandswillen der deutschen Bevölkerung schätzte die Mail gering ein. Politische Unruhen oder gar eine Revolution erwartete das Massenblatt nicht. ${ }^{403}$

Eine Gegenposition bezog der Daily Herald, der die Ruhrbesetzung verurteilte, auch wenn er gleichzeitig Verständnis für den Entente-Partner äußerte.

France was never more afraid of Germany than now when Germany is paralysed and impotent. She dreads the war of revenge. She looks with terror at the German population, far bigger than her own already, growing every year while hers dwindles. And she wonders how long it will be before the German armies come westwards again to revenge 1918, as 1918 revenged 1871, as Waterloo revenged Jena, and Jena Rossbach - and so on ad infinitum. ${ }^{404}$

Die Politik, die Paris jetzt verfolgte, werde aber genau das provozieren. ${ }^{405}$ Für Großbritannien bleibe deshalb nur die Alternative, sich von Frankreich zu lösen, auf eine allgemeine Abrüstung hinzuwirken und den Völkerbund zu stärken. ${ }^{406}$

Mit den Spitzen der britischen Arbeiterbewegung stimmte der Daily Herald darin überein, dass die Ruhrbesetzung ein vom Friedensvertrag nicht gedeckter „Akt des Krieges“ war. ${ }^{407}$ Bezeichnenderweise wurden in den Überschriften immer häufiger Anleihen aus der Militärsprache genommen. So war statt von Besetzung von Invasion sowie im Zusammenhang mit der Ausrufung des pas-

401 Ebd., 22. Januar 1923, „Hats Off To France - Her Cause Is Ours Too“.

402 Vgl. z.B. ebd., 6., 8., 12. Januar 1923.

403 Ebd., 11. Januar 1923, LA „God-Speed To France“.

404 The Daily Herald, 3. Januar 1923, LA „From The Workers’ Point Of View - What We Must Do For France“.

405 Besonders wenn Frankreich sein eigentliches Ziel erreiche, die Abtrennung des Rheinlandes und des Ruhrgebiets. Ebd.

406 The Daily Herald, 5. Januar 1923, LA „From The Workers’ Point Of View - After The Break“.

407 Vgl. die gemeinsame Resolution des „National Joint Council of the TUC General Council“, des „Executive Committee of the Labour Party“ und der „Parliamentary Labour Party“ abgedruckt in ebd., 15. Januar 1923. 
siven Widerstands und den französischen Gegenmaßnahmen von „ökonomischer Kriegführung"408, „Zermürbungskrieg“409 und „Frankreich setzt Hungerwaffe ein" 410 die Rede. Auf sprachlicher Ebene fand so eine Gleichsetzung mit dem Ersten Weltkrieg statt, nur diesmal mit vertauschten Rollen. In der Darstellung des Daily Herald war jetzt Frankreich der Aggressor und Deutschland das Opfer.

Dass der eigentliche Gefahrenherd für den Frieden in Europa nicht mehr in Berlin, sondern in Paris lag, daran hatte der Herald seit dem Amtsantritt von Poincaré keinen Zweifel gelassen. Nach den Drohungen des französischen Ministerpräsidenten gegen Deutschland im April 1922 hatte die Arbeiterzeitung ihn in Anspielung auf die Drohgebärden des wilhelminischen Reiches sogar als „Kaiser Poincaré“ betitelt. 411 Zwischen der Arbeiterzeitung und der profranzösischen Rothermere-Presse entwickelte sich nach Beginn der Ruhrbesetzung ein regelrechter Schlagabtausch. Der Pressebaron habe sich zum Agenten Poincarés gemacht und unterstütze eine Politik, die die Interessen der britischen Wirtschaft und damit auch der Arbeiter in England schade, kommentierte der Herald Rothermeres Äußerungen. ${ }^{412}$

In Kontinuität zu seiner bisherigen Kritik an der Reparationspolitik der Siegermächte hielt auch der Manchester Guardian das französische Vorgehen für einen Fehler. Nach Ansicht des stellvertretenden Chefredakteurs Charles Montague war völlig klar, dass Deutschland bankrott war. Objektive Beobachter hätten das schon 1919 erkannt, eine Anspielung auf die Vertragskritik von Keynes. Die völlig überhöhten Reparationsforderungen waren in Montagues Augen nur aus der damaligen Stimmung heraus zu erklären. ${ }^{413}$

It was an effort to utter, in terms of money, the immense accumulated anger of a halfruined Europe against those whom it felt to be the authors of its ruin - and who had already been driven from Germany by the Germans, whom they had ruined more completely. The Kaiser, it seemed, could not be hung, but a wild, hyperbolical fine, called reparation, could be inflicted on Republican Germany, as a kind of financial symbol or emblem of the hideousness of the crime of $1914 .{ }^{414}$

408 Ebd., 18. Januar 1923, „Economic Warfare In The Ruhr - Invasion Stops Industry“.

409 So der Pariser Korrespondent George Slocombe in seinem Bericht „France To Starve Germany". Ebd.

410 Schlagzeile über dem Bericht von Morgan Philips Price aus Essen über Streiks der Bergarbeiter, Einschränkungen im Zugverkehr und die Einschließung des Ruhrgebiets durch französische Truppen. The DaILY Herald, 23. Januar 1923. Slocombe sprach sogar von einer „kriegsähnlichen Expedition“ gegen eine „feindliche Bevölkerung“. THE DAILY Herald, 29. Januar 1923.

411 Richards, Taming, S.59. Als der französische Ministerpräsident im Mai schon einmal gedroht hatte, in die Ruhr einzumarschieren, hatte der Daily Herald in einem Leitartikel gewarnt: „Once again the choice is between peace and M. Poincaré.“

412 The Daily Herald, 20. Januar 1923, LA „From The Workers’ Point Of View - M. Poincaré's Press Agent".

413 The Manchester Guardian, 5. Januar 1923, LA „Failure“.

414 Ebd. 
Als tatsächliches Ziel, das Frankreich mit der Ruhrbesetzung verfolgte, vermutete der Guardian die Zersplitterung des Reiches durch eine Abtrennung des Rheinlands, was eine Gefahr für den Frieden in Europa darstellte. ${ }^{415}$ In Anbetracht der möglichen Machtverschiebungen auf dem Kontinent glaubte das liberale Blatt nicht, dass England seine Politik der „wohlwollenden Neutralität" lange werde durchhalten können. ${ }^{416}$

Die Ablehnung der französischen Exekutionspolitik in der liberalen und der linken Zeitung entsprach ihren bisherigen Positionen und war deshalb zu erwarten gewesen. Wie sehr sich das Meinungsklima in Großbritannien seit Kriegsende in Bezug auf das Weimarer Deutschland verändert hatte, verdeutlichen die Kommentare zur Ruhrbesetzung in den konservativen Qualitätszeitungen. Sowohl der Daily Telegraph als auch die Times hatten einen vorsichtigen, aber auffallenden Positionswechsel vollzogen.

Wie ausführlich dargelegt, hatten beide 1919 ernsthafte Zweifel an den innenpolitischen Veränderungen im Deutschen Reich geäußert und den Versailler Vertrag gegen Kritik verteidigt. Im Daily Telegraph lebte der alte „war time spirit“ im Januar 1923 noch einmal kurzzeitig auf. So wurden die Deutschen davor gewarnt, die britische Passivität als Unterstützung misszuverstehen. Sie hätten nach wie vor keine Freunde in England und würden auch nicht von ihren Vertragspflichten entbunden. ${ }^{417}$ Gleichzeitig wies die Zeitung auf den Schwachpunkt der französischen Politik hin, die die Reparationen als Mittel der Bestrafung Deutschlands instrumentalisiere und dabei die Zahlungsfähigkeit ihres Schuldners herabsetze. ${ }^{418}$ Die Politik, die die Alliierten bei der Implementierung des Friedensvertrags verfolgt hatten, wurde vom Telegraph insgesamt in einem neuen, kritischeren Licht gesehen. Die Siegermächte hätten es versäumt, die Anhänger der Weimarer Republik zu unterstützen, um auf diese Weise die erste deutsche Demokratie zu stabilisieren und eine Erfüllung des Vertrags zu garantieren. „The Allies never have done anything to let the Germans see that the Republic, as something apart from the empire, is worth having." ${ }^{19}$ Als die französischen Besatzer an der Ruhr auf erheblichen Widerstand stießen und sich abzeichnete, dass in naher Zukunft keine Reparationen

415 Ebd., 11. Januar 1923, LA „The Invasion Of The Ruhr“. Ideen einer Loslösung des Rheinlandes kursierten in französischen Regierungskreisen und der Presse schon seit Kriegsende. Aktivitäten in diese Richtung entfaltete vor allem der französische Hohe Kommissar in Koblenz, Tirard, während Ministerpräsident Poincaré bis zum Herbst 1923 keinerlei politische Schritte zur Gründung eines unabhängigen Rheinlandstaates unternahm. Vgl. BARIÉTY, Französische Politik, S. 14-17. Zu Entstehung und Erfolgsaussichten der separatistischen Bewegung im Rheinland siehe THEINE, Separatistische Bewegungen, S. 257-282.

416 The Manchester Guardian, 20. Januar 1923, LA „What Next?“ und 22. Januar 1923, LA „Neutrality“.

417 The Daily Telegraph, 11.Januar 1923, LA „The Occupation Of The Ruhr“ und 13.Januar 1923, LA „The Occupation Of Essen“.

418 Ebd., 5. Januar 1923, LA „Failure Of The Conference“.

419 Ebd., 9. Januar 1923. 
in nennenswertem Umfang zu erwarten waren, überwog die Sorge, dass die Situation außer Kontrolle geraten und ganz Europa in ein Chaos abdriften könnte. 420

Auf den gleichen Überlegungen basierte die Kursänderung, die in der Redaktion der Times erfolgt war. Schon im Vorfeld der Krise hatte der Außenpolitikchef Harold Williams konstruktive Vorschläge aus Paris vermisst und die französische Neigung zu einseitigem Vorgehen kritisiert. ${ }^{421}$ Außerdem machte sich nach der profranzösischen Ausrichtung unter Wickham Steed erstmals deutlich der Einfluss des wieder eingesetzten früheren Chefredakteurs Geoffrey Dawson bemerkbar, der in der französischen Politik ebenfalls keine Lösung für das Reparationsproblem sah.

Wegen der möglichen schwerwiegenden Folgen der Ruhrbesetzung stufte die Times die Tragweite der Ereignisse als ähnlich bedeutend ein wie die „Kriegserklärung 1914 oder der Abschluss des Waffenstillstands 1918““.422 In ihren Leitartikeln sagte die renommierte Zeitung - ähnlich wie der Daily Telegraph - voraus, dass es schwierig werde, mit „Bayonetten Kohle zu fördern“, und die Franzosen möglicherweise lediglich die Besatzungskosten würden erwirtschaften können. ${ }^{423}$ Williams war zudem davon überzeugt, dass eine Regelung der Reparationsfrage nicht in einer Atmosphäre des „leidenschaftlichen Antagonismus" gefunden werden konnte. ${ }^{424}$ In Richtung Deutschland stellte er klar, dass der Krieg in Großbritannien nicht vergessen sei.

The German attack, the cruel occupation of Belgium and the devastation of Eastern France are among the most vivid memories of the British people. Their grave anxiety at this moment is due to no consideration for the interests of Germany, whose past and present policy is not in the least calculated to awaken British sympathy, but to far broader reasons. 425

Damit meinte er die destabilisierende Wirkung und die mögliche Zuspitzung der Krise. Angesichts der Gefahren war sich die Times im Gegensatz zur Daily Mail sicher, dass die Mehrheit der britischen Öffentlichkeit den französischen Einmarsch in die Ruhr ablehnte. ${ }^{426}$

In weiten Teilen der britischen Presse war die Stimmung also auch ohne die Propagandaaktivitäten der Reichsregierung günstig für Deutschland. Außerdem arbeiteten die Zeit und die Umstände für die deutsche Seite, denn je länger die Ruhrbesetzung dauerte und je unübersichtlicher die Lage auch im Rest

420 Ebd., 20. Januar 1923, LA „The Situation In The Ruhr“ und 23. Januar 1923, LA „The Ruhr".

421 Williams an Huddleston in Paris, 22. August 1922, Williams Papers, Correspondence with Huddleston, TNL Archive, HW/1.

422 The Times, 6. Januar 1923.

423 Ebd., 12. Januar 1923, LA „The French In The Ruhr“.

424 Ebd., 16. Januar 1923, LA „The French Advance“.

425 Ebd.

426 Ebd., 12. Januar 1923, LA „The French In The Ruhr“. 
Deutschlands wurde, desto größer wurde das Verständnis für die Probleme des ehemaligen Kriegsgegners in den englischen Zeitungen und desto mehr wuchs das Unverständnis über das französische Vorgehen. Ein entscheidender Topos, der dem zu Grunde lag, war der der Unterlegenheit. Deutschland war in dieser Auseinandersetzung der „underdog“, der den Zwangsmitteln Frankreichs nichts Gleichwertiges entgegensetzen konnte. Der Ruhrkampf war „a simple conflict of wills between a people possessing all the means of pressure and people possessing none“.427 Symbol dafür war das Bild von schwer bewaffneten französischen Truppen, die in deutsche Städte einmarschierten, während die Bewohner ohnmächtig zusahen. ${ }^{428}$ Die Konstellation des Konflikts widersprach dem britischen Verständnis von Fairness, was zur Folge hatte, dass sich die Franzosen zwar militärisch in der stärkeren Position befanden, im Urteil vieler englischer Journalisten politisch, rechtlich und moralisch aber die schwächeren Argumente vertraten. ${ }^{429}$

Ein weiteres wichtiges Motiv war das des „Leidens“ der deutschen Zivilbevölkerung, das durch die Eskalation des Ruhrkampfes und seine ökonomischen Folgen verursacht wurde. In zahlreichen Reportagen beschrieben die englischen Korrespondenten, mit welchen zum Teil drastischen Maßnahmen die französischen Besatzungsbehörden versuchten, den passiven Widerstand zu brechen. Dazu gehörten Versammlungs- und Demonstrationsverbote sowie nächtliche Ausgangssperren. Deutsche Eisenbahner, Beamte und Bürgermeister, die die Zusammenarbeit mit den Franzosen verweigerten, wurden

427 The Daily Telegraph, 5. März 1923, LA „Events In The Ruhr“.

428 In allen Berichten über den Einmarsch der Franzosen tauchte dieses Bild in variierter Form auf. Vgl. z.B. The Times, 12. Januar 1923, „French In Essen - Strong Forces Employed - Sullen Crowds“. In diesem Sinne auch THE MANCHESTER GUARDiAN, 12. Januar 1923; The Daily Herald, 12. Januar 1923; The Daily Mail, 12. Januar 1923. Letztere konterkarierte das Bild von den Deutschen als hilflose Zuschauer bzw. Opfer durch den Vorwurf, dass Deutschland sich verhalte wie ein zahlungsunwilliger Schuldner, für seine Situation folglich selbst verantwortlich war, und sich im Übrigen 1871 gegenüber Frankreich genauso verhalten habe wie jetzt die Franzosen. The DAILY MAIL, 12. Januar 1923, LA „Germans Should Have Better Memory“ und Kolumne „How Germany Made France Pay“. Wie Krumeich herausgearbeitet hat, war die Demonstration militärischer Macht und die Härte des französischen Besatzungsregimes 1923 u.a. durch die Kriegsbesatzung der Deutschen in Frankreich und Belgien zwischen 1914 und 1918 motiviert. So war die Ruhrbesetzung also auch mentalitäts- und symbolgeschichtlich eine Spätfolge des Ersten Weltkriegs. Vgl. KRUMEICH, Ruhrkampf als Krieg, S. 9-24.

429 Die Franzosen riskierten damit nach Einschätzung des Außenpolitikchefs der Times, Harold Williams, die wirtschaftliche Zukunft und politisch den Frieden in Europa. THE Times, 16. Januar 1923, LA „The French Advance“. Der Chefredakteur des Manchester Guardian, C.P. Scott, hielt die Ruhrbesetzung für absolut illegal und damit für einen Akt der Gewalt. Scott an Sir George Arthur, 19. Juni 1923, Scott Papers, General Correspondence, MGA, Box 336/88. Hier lag nach Einschätzung von Gedye auch der Grund, warum die französische Propaganda bei den britischen Korrespondenten einen so schweren Stand hatte. GEDYE, Revolver-Republik, S. 91. 
mitsamt ihren Familien aus dem Ruhrgebiet ausgewiesen. ${ }^{430}$ Städte und Gemeinden, in denen Sabotageakte gegen die Besatzungsmacht verübt wurden, mussten mit hohen Geldstrafen rechnen. Trotzdem häuften sich Anschläge auf Eisenbahnlinien, die nach dem Streik des deutschen Bahnpersonals von den Franzosen bedient werden mussten. ${ }^{431}$ Die Besatzungsbehörde drohte daraufhin Saboteuren mit der Todesstrafe. ${ }^{432}$ Manager und Fabrikdirektoren wurden von französischen Militärgerichten wegen mangelnder Kooperation zu hohen Haftstrafen verurteilt. ${ }^{433}$ Zwischen deutschen Zivilisten und französischen Soldaten eskalierte die Gewalt und es kam immer wieder zu Zusammenstößen, bei denen oft genug auch Tote zu verzeichnen waren. ${ }^{434}$

Um das Ruhrgebiet zog die französische Besatzungsmacht außerdem eine Zollbarriere, was einer wirtschaftlichen Abtrennung vom restlichen Reichsgebiet gleichkam. ${ }^{435}$ Die Folge waren wachsende Arbeitslosigkeit, Versorgungsengpässe und steigende Preise. ${ }^{436}$ Die Finanzierung des Ruhrkampfes über die Notenpresse heizte zudem die Inflation an, was zu einem weiteren Kaufkraftverlust führte und die Verarmung der Bevölkerung beschleu-

430 Vgl. u.a. The Daily Herald, 31. Januar 1923; The Times, 5., 9., 10. Februar 1923 und 31. März 1923; The Daily MaIl, 31. März 1923. Weil sich in Essen die Polizisten weigerten, vor französischen Soldaten zu salutieren, wurde die gesamte Polizei ausgewiesen, was die Kriminalitätsrate sprunghaft ansteigen ließ. ThE TıMES, 14. März 1923.

431 Der britische Vizekonsul in Essen berichtete im Februar, dass der Eisenbahnverkehr praktisch zum Erliegen gekommen war, dass die Telefon- und Telegraphenverbindungen immer wieder unterbrochen und im gesamten besetzten Gebiet alle Bürgermeister bis auf vier entfernt worden waren. CARSTEN, Britain and Weimar, S. 131.

432 The Daily Telegraph, 3. März 1923. Der deutsche Nationalist Albert Leo Schlageter wurde wegen Sabotage am 25. Mai von den Franzosen hingerichtet, was ihn in nationalistischen Kreisen zu einem Märtyrer werden ließ. Wie der Guardian im Juni berichtete, hatte sich um ihn ein regelrechter Kult entwickelt. The Manchester GuARdian, 20. Juni 1923.

433 The Daily Herald, 19. Januar 1923.

434 The Daily Telegraph, 12. und 13. März 1923; The Manchester Guardian, 14. Juni 1923. Der Vorfall am Ostersamstag auf dem Werksgelände von Krupp war einer der schlimmsten dieser Art. Nach der französischen Darstellung sahen sich die Soldaten, die Automobile requirieren wollten, durch protestierende Arbeiter bedroht und feuerten in die Menge. Die Bilanz waren 13 Tote und 30 Verwundete. The Times, 3. April 1923. Siehe auch Zechlin, Pressechef, S. 45. Die Franzosen schoben die Verantwortung den Direktoren von Krupp zu, die die Menge zum Angriff auf die Soldaten angeheizt hätten, und ein Militärtribunal verurteilte sie zu 15 Jahren Gefängnis. The Times, 9. Mai 1923. Die französische Lesart der Ereignisse sorgte bei den englischen Korrespondenten für Empörung. Gedye und Thomas berichteten übereinstimmend, dass die Soldaten die Nerven verloren und „kalten Blutes“ auf die Arbeiter geschossen hatten. Der Prozess gegen Krupp und seine Direktoren war in ihren Augen eine „fantastische Parodie“ von Gerechtigkeit, da Beweise fehlten und das Urteil vorher feststand. Thomas, Traveller, S. 207 und Gedye, Revolver-Republik, S. 127 und 130f. Eine genaue Rekonstruktion der Ereignisse und des anschließenden Prozesses ist zu finden bei WisOTZKY, Karsamstag, S. 265-287.

435 The Times, 17. Januar 1923.

436 Ebd., 13. Februar 1923. 
nigte. ${ }^{437}$ Die englischen Journalisten wie William Beach Thomas empfanden die Situation als „Alptraum“.

Before I left Germany in the late summer [...] the mark was not so much as one thousand millionth part of a Pound. Most of the Ruhr magnates from Krupp to Bohlen downwards were undergoing terms of up to fifteen years' imprisonment. At least twenty-six thousand railwaymen were evicted and banished from the Rhine. Railways were either littered with wreckage or rusted with disuse. [...] Death and destruction were daily events. ${ }^{438}$

Die Berichte über die Not vieler Menschen lösten in Großbritannien eine Welle der Hilfsbereitschaft aus. Zahlreiche Organisationen mit den unterschiedlichsten politischen Verbindungen wurden aktiv, darunter der Save the Children Fund und der German Distress Relief Fund, in denen führende Mitglieder der Arbeiterbewegung den Ton angaben. ${ }^{439}$ Die Labour Party selbst sammelte Geld für die deutschen Arbeiterorganisationen. ${ }^{440}$ Das University Committee of the Imperial War Relief Fund mit guten Kontakten in der Londoner Finanzwelt bemühte sich in kirchlichen, literarischen und akademischen Kreisen erfolgreich um Unterstützung, u.a. für verarmte deutsche Studenten und Dozenten. $^{441}$

Die Zuspitzung der Lage in Deutschland, die gekennzeichnet war durch das um sich greifende wirtschaftliche Chaos, den wieder aufkeimenden Nationalismus sowie die verstärkte Agitation von Kommunisten und Rechtsextremisten, bestätigte die Kritiker der Ruhrbesetzung in den Londoner Redaktionen. Es war zwar offensichtlich, dass die Deutschen sich mit ihrer Strategie des passiven Widerstands massiv selbst schadeten. ${ }^{442} \mathrm{Im}$ Gegenzug erreichten aber auch die Franzosen ihr Ziel, Reparationen in Form von Kohle-, Koks- und Holzlieferungen in größerem Umfang zu erzwingen, nicht. Insofern war die

437 Die Hohe Kommission für das Rheinland berichtete, dass es im besetzten Gebiet im Sommer kaum noch Kartoffeln zu kaufen gab. Die Versorgungslage verschärfte sich im Herbst und Winter auch im restlichen Reichsgebiet so dramatisch, dass Plünderungen von Lebensmittelgeschäften und Fahrzeugen alltäglich waren. CARSTEN, Britain and Weimar, S. 145-147.

438 Thomas, Traveller, S. 202. Ebenso GibBs, Pageant, S. 339-341.

439 Im Komitee des „German Distress Relief Fund“ saßen u.a. Arthur Ponsonby und Bertrand Russell. Williamson, Ruhr Crisis, S. 79.

440 Ebd.

441 Unterstützung erhielt die Organisation z.B. vom Erzbischof von Canterbury und Lord Robert Cecil. Ebd., S.78. Das diplomatische Korps in Berlin organisierte unter der Schirmherrschaft der Amerikanischen Botschaft einen Wohltätigkeitsball. Die Einnahmen gingen an Suppenküchen der Hungerhilfe. D’ABERNON, Red Cross, Eintrag vom 9. Dezember 1923, S. 125. Die Gattin des britischen Botschafters beschrieb auch die Auswirkungen der Inflation wie wachsenden Hunger und zunehmende Plünderungen.

442 Der Telegraph und der Guardian hatten wegen der zu erwartenden hohen finanziellen und sozialen Kosten schon im März vorausgesagt, dass der Widerstand irgendwann aufgegeben werden musste. The Daily Telegraph, 3. März 1923; The Manchester GuarDIAN, 19. März 1923. 
Ruhrbesetzung der von vielen in England erwartete Fehlschlag. Der Manchester Guardian sprach von einer "Riesenverschwendung französischen Geldes und einer noch größeren Verschwendung deutscher Ressourcen“..443

Lediglich die Daily Mail blieb konsequent bei ihrer profranzösischen Linie und zeigte sich von den Ereignissen unbeeindruckt. Rothermere hielt daran fest, dass das französische Vorgehen erfolgreich war und von einem Sieg gekrönt werden würde. ${ }^{444}$ Auch Interventionen von Seiten der Politik konnten ihn nicht umstimmen. Nach einem Treffen des Pressebarons mit Churchill, Lord Birkenhead und Lloyd George notierte der ehemalige Premierminister, Rothermere sei „just widely pro-French over the Ruhr“. ${ }^{445}$ In der Mail ließ der Verleger weiter erklären, dass Deutschland zur Zahlung der Reparationen in voller Höhe fähig war. ${ }^{446}$ Berichte anderer Zeitungen über die Notlage der Bevölkerung in den besetzten Gebieten, die infolge der Einschränkungen im Zugverkehr z.B. mit Engpässen bei der Lebensmittelversorgung zu kämpfen hatte, tat das Massenblatt als „Propagandageschrei aus Berlin“ ab.447

Als die Reichsregierung wegen der explodierenden Kosten und der desolaten Finanzlage schließlich Ende September 1923 den Abbruch des „passiven Widerstands" erklärte, fiel der Kommentar in der Daily Mail entsprechend euphorisch aus. Die Entscheidung Berlins sei ein Triumph für Frankreich und für dessen Entschlossenheit, die Deutschen zur Einhaltung des Versailler Vertrags $\mathrm{zu}$ zwingen. Die Voraussagen der anderen Zeitungen vom Januar, allen voran der Times, dass die Besetzung in einem "Desaster" enden werde, seien damit widerlegt. Auf die negativen Folgen der französischen Besatzungspolitik, wie die zahlreichen Todesopfer auf beiden Seiten, ging die Mail nicht ein, sondern behauptete, die Franzosen hätten einen Sieg „ohne Blutvergießen“ erreicht. ${ }^{448}$ Erneut nahm das Massenblatt außerdem für sich in Anspruch, mit seiner Parteinahme für die Franzosen stellvertretend für die gesamte britische Nation

443 The Manchester Guardian, 12. Juni 1923, LA „The Accepted Time“.

444 Rothermere an Beaverbrook, 26. April 1923, Beaverbrook Papers, Correspondence with Rothermere, HLRO, BBK/C/283a.

445 Lloyd George hoffte trotzdem, dass das Gespräch „may produce a more friendly atmosphere in his papers". MORGAN (Hrsg.), Family Letters, S. 200.

446 The Daily Mail, 14. März 1923.

447 Ebd., 25. Juni 1923. Der Daily Telegraph beispielsweise hatte nur wenige Tage zuvor über die Schwierigkeiten bei der Anlieferung von Nahrungsmitteln berichtet, da kaum noch Züge zwischen besetztem und unbesetztem Gebiet fuhren. The Daily Telegraph, 20. Juni 1923.

448 Ebd., 26. September 1923, LA „The Triumph Of France“. Im Original „a bloodless victory“. Zur Bestätigung ihres Standpunkts, dass Deutschland trotz Inflation und der Abtrennung des Ruhrgebiets vom übrigen Reich die Wirtschaftskraft besaß, Reparationen zu zahlen, druckte die Zeitung beispielsweise einen Augenzeugenbericht des konservativen Unterhausabgeordneten Charles Foxcroft. Darin verglich der Politiker die vom Krieg zerstörten Landstriche und Städte im Osten Frankreichs mit den unzerstörten Feldern, Wäldern und Industriegebieten im Rheinland. The DaiLY MaIL, 11. September 1923, „What A Contrast! - Defeated Germany And Victorious France“. 
zu sprechen und behauptete, die „pro-Germans“ in der Presse, im Parlament und im Kabinett hätten das Gespür für die Stimmung in der britischen Bevölkerung verloren. ${ }^{449}$

Die Angriffe des Rothermere-Blattes, die auf alle zielten, die mit der deutschen Seite sympathisierten, parierte der Daily Herald mit der Abwandlung eines Zitats des Pressebarons. Statt „Hats off to France“ betitelte die Arbeiterzeitung eine Solidaritätsadresse an die deutschen Arbeiter im Ruhrgebiet mit der Schlagzeile „Hats Off To Our Brave Ruhr Comrades!“ ${ }^{450}$ Diese hätten unglaublichen Mut gezeigt in einem Kampf, in dem jetzt „Brutalität über Hilflosigkeit“ gesiegt habe. Der Vorwurf, „pro-deutsch“ zu sein, wurde als antiquiert zurückgewiesen. Der Herald setze sich für jeden ein, der ungerecht behandelt werde. ${ }^{451}$

Die Qualitätszeitungen dagegen kamen zu dem einhelligen Ergebnis, dass die Ruhrbesetzung die damit von Anfang an verbundenen Risiken nicht wert gewesen war. ${ }^{452}$ In Anbetracht der wirtschaftlichen Destabilisierung sowie des drohenden politischen Auseinanderbrechens Deutschlands stimmten die Leitartikler darin überein, dass man von dem erklärten Ziel der Franzosen, die Begleichung der Kriegsschulden zu erreichen, weiter denn je entfernt war. Die Interpretation, dass die Deutschen „kapituliert“ und Frankreich „gesiegt“ habe, konnte die Times folglich nicht nachvollziehen. „if, as we have always believed, the German power to pay has been steadily reduced by the Franco-Belgian occupation, the ,victory' must certainly be classed as Pyrrhic." 453

Ein Vergleich der Positionen in der Presse zum Vorgehen Frankreichs bzw. zur Rolle Deutschlands während der Ruhrkrise mit denen in der britischen Politik ergibt eine weitgehende Übereinstimmung zwischen den Vertretern der unterschiedlichen außenpolitischen Denkschulen und den ihnen nahe stehenden Zeitungen. So verurteilten die Unterhausabgeordneten der Labour Party den Alleingang des Entente-Partners und äußerten durchweg Verständnis für die Deutschen. Ramsay MacDonald kritisierte die gesamte Reparationspolitik als Bestrafungsaktion. ${ }^{45}$ Die MPs Buxton und Shaw, die beide an der Ruhr ge-

449 Ebd., 24.September 1923, LA „Why Our Pro-Germans Are Angry“. Die Gegenseite bestritt das. Dawson war seinerseits davon überzeugt, dass 90 Prozent der Engländer die Ruhrbesetzung ablehnten. The Times, 14. Mai 1923, LA „The British Reply“.

450 The Daily Herald, 27. September 1923, LA „From The Workers’ Point Of View - Hats Off To Our Brave Ruhr Comrades“.

451 Mit der Feststellung, Frankreich verfahre nach dem Prinzip „might is right“, setzte der Kommentator zudem erneut die französische mit der wilhelminischen Außenpolitik gleich. Ebd.

452 The Times, 26. September 1923, LA „The German Decision“; The Manchester GuarDian, 26. September 1923, LA „The Last Hours“; The Daily TelegraPH, 27. September 1923, LA „Germany In Defeat“.

453 The Times, 26. September 1923, LA „The German Decision“.

454 PD/C, V, 160, Spn. 23f., 13. Februar 1923. 
wesen waren, warnten vor den sozialen Folgen und dem Widerstandsgeist bzw. dem Hass, den die Franzosen provozierten. ${ }^{455}$ Die Annahme, dass die Deutschen nur die Sprache der Gewalt verstünden, wies Shaw zurück. ${ }^{456}$ Sein Kollege Clynes verurteilte die Ruhrbesetzung als einen Akt der Aggression, der genauso falsch sei, wie der deutsche Angriff auf Belgien und Frankreich $1914 .{ }^{457}$

Asquith für die Liberalen und Bonar Law als Premierminister der konservativen Regierung nahmen moderatere Positionen ein. Asquith betonte einerseits die moralische Verantwortung Deutschlands für den Krieg und seine Folgen, sprach sich andererseits aber dafür aus, dass im Zentrum der Reparationsfrage Deutschlands Zahlungsfähigkeit stehen müsse und begrüßte die Weigerung der britischen Regierung, an dem „Abenteuer in der Ruhr“ teilzunehmen. ${ }^{458}$ Bonar Law äußerte zwar Verständnis für die Furcht Frankreichs vor Deutschland, die der eigentliche Grund für den Einmarsch gewesen sei. Gleichzeitig verwies er aber darauf, dass von Deutschland keine akute Gefahr ausgehe und verteidigte dessen Politik der „wohlwollenden Neutralität“.459

Auf den hinteren Bänken der Konservativen herrschte dagegen offene Sympathie für Frankreich. Die Abgeordneten Davison und Remer etwa waren der Auffassung, dass erst die Divergenzen zwischen Großbritannien und Frankreich die Deutschen zu ihrer Verweigerungshaltung angestachelt und ihnen die Möglichkeit eröffnet hatte, die Alliierten gegeneinander auszuspielen. ${ }^{460}$ Wenn die Siegermächte zusammenstehen würden, dann hätte Deutschland gar keine andere Wahl, als die Reparationen zu bezahlen, so Remer. ${ }^{461}$

In prägnanter Form sind hier alle Argumente der verschiedenen politischen Richtungen anzutreffen: die imperialistische Rechte, die bedingungslos zu Frankreich stand, Deutschland nach wie vor als Täter, sprich Verantwortlichen für den Krieg sah und in der Daily Mail einen Verbündeten fand; die gemäßigten Konservativen und Liberalen, für die der Versailler Vertrag Grundlage der Nachkriegsordnung war, die seine Defekte aber anerkannten, eine Revision anstrebten - wenn möglich im Konsens mit Frankreich und Deutschland - und

455 Ebd., Spn. 46-49 und 66-68. Buxton hatte über seine Erlebnisse im Daily Herald Auskunft gegeben. Charles Roden Buxton, „Among The Ruhr Workers“, The Daily Herald, 31.Januar 1923. Es waren fast ausschließlich Vertreter der britischen Arbeiterbewegung, die in dieser Zeit Deutschland besuchten, um sich ein eigenes Bild zu machen. Vgl. THOMAs, Traveller, S.222.

456 „The feeling that the only way to make the Germans understand is to treat them ruthlessly and cruelly is a theory which is absolutely without foundation", so Shaw wörtlich. PD/C, 5 (160), Sp. 68.

457 Ebd., Sp. 366.

458 Ebd., Spn. 30-33.

459 Ebd., Spn. $43 \mathrm{f}$.

460 Ebd., Spn. 58-60 und 79f.

461 Wörtlich erklärte er: „I believe the German defiance will only last while we stand aloof or are lukewarm in our attitude in regard to France." Ebd., Sp. 60. 
die von der Times, dem Daily Telegraph und dem Manchester Guardian publizistisch unterstützt wurden; und schließlich die Linke, die Deutschland geläutert und jetzt in der Rolle des Opfers der französischen Politik sah, die von Großbritannien eine unparteiische Vermittlung forderte und deren Sprachrohr der Daily Herald war.

An den Aktivitäten hinter den Kulissen lässt sich darüber hinaus ablesen, wie eng die Verzahnung zwischen Journalisten und Politikern in dieser Phase war und wie die Kommunikation zwischen den Akteuren ablief. Die Erkenntnis, dass die gesamte Situation festgefahren war und sich die britische Außenpolitik in einer Sackgasse befand, in der sie nur wenig Spielraum für eigene Initiativen hatte, veranlasste die Chefredakteure des Guardian, Scott, und der Times, Dawson, sich bei der Suche nach Lösungen stärker zu engagieren, was sie im Diskurs mit der politischen Spitze taten.

Scott beschäftigte vor allem die Frage nach der Rechtmäßigkeit der Ruhrbesetzung. Schon einen Monat vor deren Beginn hatte er mit Premierminister Bonar Law über die rechtliche Grundlage für den sich abzeichnenden französischen Alleingang gesprochen. ${ }^{462}$ Dabei hatte sich angedeutet, dass der Regierungschef bereit war, einen Einmarsch in die Ruhr als Sanktion für den „freiwilligen Verzug“ Deutschlands bei den Reparationslieferungen hinzunehmen. Scott hatte entschieden widersprochen, dass der Verzug - wie von den Franzosen behauptet - freiwillig war, und darauf hingewiesen, dass es für die Deutschen wegen des fehlenden „limits“ keinen Anreiz gab, die alliierten Reparationsforderungen zu erfüllen. ${ }^{463}$ Scott war der Ansicht, dass eine Besetzung der Ruhr vom Versailler Vertrag keinesfalls gedeckt war, worin er vom britischen Rechtsexperten bei der Reparationskommission, John Fischer Williams, bestärkt wurde. ${ }^{464}$ Gemeinsam drängten sie den deutschen Botschafter Sthamer Anfang März 1923, die Legalität der Ruhrbesetzung kontinuierlich anzufechten, weil das Gespür für Recht und Unrecht in England besonders ausgeprägt sei. Auf diese Weise ließe sich die Position Frank-

462 Scott Papers, Political Diaries, Eintrag vom 6. Dezember 1922, MGA, Box 134.

463 Letzterem stimmte Bonar Law zu. Ebd.

464 Beide standen in brieflichem Kontakt, so dass Scott über die Vorgänge in der Kommission aus erster Hand informiert war. Vgl. Fischer Williams an Scott, 24. und 27. Januar 1923, Scott Papers, General Correspondence, MGA, Box 336/62. Mehrere Artikel im Manchester Guardian, in denen schon im Mai 1922 die Legalität von einseitig durch Frankreich verhängten Sanktionen gegen Deutschland bestritten wurde, hatten eine Diskussion darüber im Foreign Office ausgelöst. Crowe bezweifelte, ob die Position Frankreichs mit rechtlichen Argumenten zu erschüttern sei, da die Artikel des Versailler Vertrags unterschiedliche Interpretationen zuließen und schlug vor, stattdessen auf die Einhaltung des französischen Versprechens zu pochen, nicht allein zu handeln. Notiz Crowes, 21. Mai 1922, PRO, FO 371/7477, C7548/99/18. Headlam-Morley argumentierte hingegen im Sinne des Guardian, nämlich dass sich aus dem Text und seiner Interpretation durch die Siegermächte auf den verschiedenen interalliierten Konferenzen kein Recht darauf ableiten ließ. Notiz Headlam-Morleys, 24. Mai 1922, PRO, FO, 371/7477, C7873/99/18. 
reichs in der britischen Öffentlichkeit erschüttern. ${ }^{465}$ Scott bemühte sich außerdem darum, dass dieser Punkt in den Debatten im Unterhaus zur Sprache kam. 466

Für Dawson lag das Problem in der Vermengung der ökonomischen mit den sicherheitspolitischen Aspekten der Versailler Friedensordnung. In seiner Analyse diente die Ruhrbesetzung erst in zweiter Linie dazu, Reparationen zu erzwingen. Viel wichtiger war das Streben Frankreichs nach Sicherheit vor einem erneuten deutschen Angriff. Dazu wollte Paris offensichtlich das Rheinland und die Ruhr vom übrigen Reichsgebiet trennen. Um das zu verhindern und Frankreich gleichzeitig von seiner Furcht vor seinem östlichen Nachbarn zu befreien, müsse England eine Beistandsgarantie für Paris abgeben. ${ }^{467}$ In diesem Sinne drängte die Times in ihren Leitartikeln auf eine aktivere britische Politik, die einen entsprechenden Pakt anbieten sollte. ${ }^{468}$ Dawson verlangte außerdem, die Zahlungsfähigkeit Deutschlands durch eine unabhängige Kommission untersuchen zu lassen, und von den Deutschen, dass sie ihre Reparationspflicht grundsätzlich anerkannten. ${ }^{469}$ Auf diese Weise, hoffte er, war eine Trennung der Sicherheits- von der Reparationsfrage und damit die Beendigung der Krise möglich. Diesen Lösungsvorschlag erörterte der Chefredakteur bei seinen regelmäßigen Gesprächen mit Premierminister Bonar Law und dessen Nachfolger Baldwin. 470

465 Scott Papers, Memoranda Recording Conversations with Prominent Figures 1911-28, Eintrag vom 10. März 1923, BL, SADM 50907. Die beiden drängten Sthamer außerdem, dass die Reichsregierung ihre Bereitschaft erklären müsse, jede vernünftige Summe zu bezahlen, von der ein unparteiisches Tribunal festgestellt hatte, dass Deutschland in der Lage war, sie zu begleichen.

466 Er machte u.a. Sir John Simon darauf aufmerksam. Der Abgeordnete der liberalen Partei und ehemalige Innenminister antwortete ihm, dass sein Parlamentskollege James Butler in der Debatte am 19. Februar eine entsprechende Anfrage an die Regierung gestellt hatte, Bonar Law eine Antwort aber schuldig geblieben sei. Simon an Scott, 2. März 1923, Scott Papers, General Correspondence, MGA, Box 336/72.

467 Dawson an den Rechtsanwalt Newton Rowell, Toronto, 13. Februar 1923, Dawson Papers, General Correspondence, BLO, MS Dawson 69. Ein solcher Beistandspakt war seit dem Nichtzustandekommen der Sicherheitsgarantie für Frankreich 1919 infolge der ausbleibenden Ratifikation des Versailler Vertrags und der Völkerbundsakte durch die USA immer wieder im Gespräch. Verhandlungen über eine formelle Allianz zwischen Großbritannien und Frankreich, die Anfang Dezember 1921 begonnen worden waren, scheiterten auf der Konferenz von Cannes im Januar 1922. Im Foreign Office wurde aber weiter darüber diskutiert, in welcher Form Großbritannien die Sicherheit Frankreichs bei einem deutschen Angriff garantieren könne. Vgl. ORDE, International Security, S.12-19 und 30-32.

468 Vgl. u.a. The Times, 20.Februar 1923, LA „The British Task“ und 14. März 1923, LA „The European Crisis“.

469 Ebd., 14. Mai 1923, LA „The British Reply“.

470 Allein für den Januar 1923 sind fünf Treffen mit Bonar Law belegt. WRENCH, Dawson, S. 215. Mit Baldwin sprach er am 17. und 20. Juni sowie am 22. September. Vgl. die Aufzeichnungen darüber in Dawson Papers, General Correspondence, BLO, MS Dawson 27 und 70. 
Dass die Verantwortlichen der renommierten Zeitung glaubten, einen konstruktiven Beitrag leisten zu können, zeigen Aussagen des Außenpolitikchefs der Times und ihres Berliner Korrespondenten. Williams beschrieb die Situation in London im Mai 1923 folgendermaßen:

People here generally have no wish to break with France and yet how to assert ourselves as we ought to assert ourselves and not break with France, is a problem no one knows quite how to solve. [...] Our policy in the paper is to try our level best to keep the door open [für Verhandlungen, der Verf.] so that our country may still have room to act. 471

In der deutschen Hauptstadt war Daniels zu dem Schluss gekommen, dass es an der Zeit war, „when we should do everything that will help to get the reparation question out of the way." ${ }^{472}$ Die Times-Redaktion sah sich selbst folglich gegenüber der Politik in einer beratenden Rolle, während sie sich gleichzeitig darum bemühte, der Regierung den Handlungsspielraum in der öffentlichen Debatte zu erhalten. Das tat sie, indem sie in ihren Kommentaren immer wieder darauf drängte, das Angebot, das Berlin Anfang Juni zur Lösung der Reparationsfrage unterbreitet hatte, als Grundlage für Verhandlungen anzunehmen, und auf der anderen Seite die Bedingungen, die Paris für den Beginn von Gesprächen aufstellte, kritisierte. ${ }^{473}$

Die kompromisslose Haltung der Regierung Poincaré, die auf einem sofortigen Ende des passiven Widerstands beharrte, die eine Untersuchung der deutschen Zahlungsfähigkeit durch ein unabhängiges Gremium ablehnte und eine Besetzung der Ruhr bis zur Begleichung der Reparationsschuld erwog, traf auch in den anderen Zeitungen auf wachsende Ungeduld. ${ }^{474}$ Die Ablehnung der französischen Politik gründete sich nicht nur auf ihre Inflexibilität, sondern außerdem darauf, dass die Ruhrbesetzung im Alleingang ohne Rücksicht auf den britischen Partner erfolgt war, dass die eingesetzten Mittel in keinem Verhältnis zur Verfehlung der deutschen Seite standen und nie eine Aussicht auf Erfolg bestanden hatte. So verfestigte sich der Eindruck, dass, ähnlich wie Deutschland vor dem Krieg, jetzt Frankreich in Wirklichkeit nach der „iron

471 Williams an Daniels, 9. Mai 1923, Williams Papers, Correspondence with Daniels, TNL Archive, HW/1.

472 Daniels an den Foreign News Editor Ralph Deakin, 9. Mai 1923, Correspondence between Ralph Deakin and Harold G. Daniels, TNL Archive, TT/FN/1/RD/1.

473 So z.B. The Times, 9. Juni 1923, LA „A Basis For A Settlement“ und 11.Juni 1923, LA „Towards A Conference“ und 21.Juni 1923, LA „Warnings“. Ein erstes deutsches Angebot Anfang Mai hatte eine so geringe Gesamtsumme enthalten, dass die britische Regierung es als völlig ungenügend zurückwies. Daraufhin hatte die Reichsregierung am 7. Juni 1923 eine weitere Note an die Alliierten geschickt, die umfassende Garantien für künftige Reparationsleistungen vorsah. BERGMANN, Reparationen, S. 239-241.

474 Vgl. u.a. The Daily Herald, 8. Juni 1923, LA „The Latest ,Note“; The Manchester Guardian, 8. Juni 1923, LA „The German Proposal“; The Daily Telegraph, 9. Juni 1923, LA "Germany's Memorandum“. Die Daily Mail stufte die deutsche Offerte als „bloße Propaganda“ ein. The Daily Mail, 9.Juni 1923, LA „The New German ,Offer“". 
mastery of Europe“ 475 strebte und den Friedensvertrag ausnutzte, um seine „imperialistischen Ziele“ $476 \mathrm{zu}$ erreichen.

Vor diesem Hintergrund wanderten die Sympathien fast automatisch auf die Seite des Schwächeren, in diesem Fall Deutschland. Der Wandel in der Wahrnehmung wurde durch die hier untersuchten Zeitungen - mit Ausnahme der Daily Mail - entweder explizit oder implizit kommuniziert, wie die angeführten Beispiele verdeutlichen, und wirkte sich auch auf die Beurteilung des Verhaltens der Deutschen in Bezug auf den Versailler Vertrag aus. War vor allem im konservativen Meinungsspektrum bis 1922 das Motiv von den „Betrügern“ und „Tricksern“ dominant, die mit allen Mitteln versuchten, um eine Erfüllung der Bestimmungen herumzukommen, so wurde dies zunehmend überlagert durch die Ansicht, dass der Vertrag eine Reihe ungerechtfertigter Härten enthielt und eine buchstabengetreue Umsetzung nicht möglich war, was insbesondere auf die finanziellen Regelungen zutraf. Es erfolgte also eine Annäherung der Kritiker aus liberalen und sozialistischen Kreisen mit den gemäßigten Konservativen.

Ein dabei nicht zu unterschätzender Faktor waren die wirtschaftlichen Schwierigkeiten Großbritanniens, die zu der Erkenntnis geführt hatten, dass eine Erholung im eigenen Land nur dann möglich war, wenn sich die ökonomischen Verhältnisse in Deutschland verbesserten. Unter diesem Aspekt hatte sich die bisherige Reparationspolitik als untauglich erwiesen. Der Gleichklang, der in diesem Punkt zwischen weiten Teilen der Presse und der Politik herrschte, ist ein Beweis für die engen Beziehungen beider Sphären. Zugleich ist er ein Hinweis darauf, dass sich das britische Urteil über Deutschland in dieser Phase stark an den eigenen nationalen Interessen orientierte und Veränderungen von der politischen bzw. publizistischen Führungsschicht ausgingen, eine These, die sich mit der Analyse deutscher Diplomaten im Auswärtigen Amt deckt. ${ }^{477}$

Diese führenden Gruppen haben durchweg heute ihre Bitterkeit Deutschland gegenüber abgestreift. Sie sind erschüttert durch die hoffnungslose Wirtschaftslage und geben Frankreich durchweg die Schuld. Man darf nicht sagen, daß eine besondere Freundschaft für Deutschland als solches existiert, aber man ist sich klar, daß die Gesundung der ganzen Welt unmöglich wird - und besonders für den britischen Handel - wenn Deutschland mehr und mehr in den Abgrund gleitet. ${ }^{478}$

475 ThOMAs, Traveller, S. 222.

476 GEDYE, Reconciliation, S. 124.

477 Der Schlussfolgerung Müllers, dass der Anteil der deutschen Propaganda an diesem Wandel eher gering einzuschätzen sei, ist deshalb zuzustimmen. MÜLLER, Auswärtige Pressepolitik, S. 143.

478 Aufzeichnung des Ministerialdirektors von Schubert: „Politische Lage und Stimmung in England“, 16. Oktober 1923, ADAP, A, 8, 191, S. 493. In diesem Sinne fiel Ende Oktober auch der Reisebericht des ehemaligen deutschen Botschafters in London, Graf von Metternich, aus: „Was mir besonders auffiel, war die starke Verstimmung, zum Teil Abneigung gegen die Franzosen. England ist ruhe- und friedensbedürftig. Die Erholung Europas, von den Engländern als notwendig für ihre eigene Erholung empfunden, wird von den Franzosen verhindert. Diese sind die Ruhestörer." Schreiben Metternichs an von Schubert, 29. Oktober 1923, zit. nach MüllER, Auswärtige Pressepolitik, S. 142. 
Trotz der Verschiebung hin zu einer positiveren Beurteilung verschwanden die negativen Aspekte des Deutschlandbildes keineswegs aus dem britischen Bewusstsein. Ausdruck dafür war zum einen die Sorge über die im Zuge des Ruhrkampfes verstärkt auftretenden nationalistischen Extremisten auf der politischen Rechten im Deutschen Reich. ${ }^{479}$ Zum anderen blieb eine deutliche Skepsis gegenüber der Verlässlichkeit und den Methoden deutscher Außenpolitik, wofür nicht nur die Konflikte um die Reparationen, sondern auch die Differenzen über die militärischen Bestimmungen des Versailler Vertrags ein Beispiel sind.

\subsubsection{Nur 100000 Mann? Abrüstung und gebeime Aufrüstung Deutschlands}

Die im Friedensvertrag vorgesehene weitgehende Entwaffnung Deutschlands war für die britische Wahrnehmung insofern wichtig, als sie von hohem Symbolgehalt war. Die Einhaltung der Rüstungsbegrenzungen war sowohl ein Prüfstein dafür, ob die Deutschen tatsächlich mit dem Militarismus gebrochen hatten, als auch ein Gradmesser, inwieweit vom Deutschem Reich weiterhin eine potenzielle Gefahr ausging. Von besonderer Bedeutung war die Abrüstung außerdem, weil sich daran erkennen ließ, welchen Einfluss die preußische Offizierskaste, die in britischen Augen hauptverantwortlich für den Krieg war, auf die Politik der Weimarer Republik hatte. Diese Parameter bestimmten die Berichterstattung der englischen Zeitungen und das Urteil innerhalb der britischen Regierung gleichermaßen. Das Problem bestand darin, dass sich bei einer genaueren Betrachtung ein gemischtes Bild ergab. Auf der einen Seite rekrutierte sich die neue Reichswehr aus Soldaten der alten kaiserlichen Armee und es gab offensichtliche Verstöße gegen die Entwaffnungsbestimmungen des Versailler Vertrags. Andererseits war Deutschland ohne schwere Waffen und mit seiner geringen Truppenstärke nicht mehr in der Lage, einen Krieg zu führen. ${ }^{480}$ Hinzu kam, dass die Reichswehr innenpolitisch eigentlich eine stabilisierende Funktion erfüllte. In Großbritannien gab es jedoch schon früh Zwei-

479 Vgl. die Berichte über Geheimbünde wie die „Organisation Rossbach“ oder die Agitation der NSDAP Hitlers in Bayern. The Daily Telegraph, 2. März 1923; The Manchester Guardian, 27. Juni 1923; The Daily Herald, 29. September 1923. Siehe auch Teil II, Kapitel 2.5.3.

480 Die Verifizierung der tatsächlichen Zahl des militärisch geschulten Personals verursachte erhebliche Schwierigkeiten, was neben den Verschleierungsversuchen der deutschen Stellen auch an der Vielzahl der bewaffneten Organisationen lag. Neben der Reichswehr, die nach allgemeinen Schätzungen Anfang 1920 noch 400000 Mann stark war, existierten die so genannten Zeitfreiwilligen, die als Reserve dienten, die kasernierte Sicherheitspolizei, die dem Reichsinnenministerium unterstellt war, und die Einwohnerwehren, bewaffnete Verbände, die sich als Reaktion auf die bolschewistische Gefahr gegründet hatten und politisch rechts standen. Philips Price berichtete am 4. Februar $1920 \mathrm{im}$ Daily Herald, dass insgesamt 1670000 Mann in Deutschland unter Waffen standen oder militärisches Training absolvierten. PriCE, Dispatches, S. 66. 
fel, ob 100000 Mann für die Aufrechterhaltung von Ruhe und Ordnung ausreichten. ${ }^{481}$

Über den Fortgang der deutschen Abrüstungsbemühungen kristallisierten sich in den folgenden Jahren divergierende Ansichten innerhalb der britischen Administration heraus. Das Kriegsministerium war der Auffassung, dass die Auflagen bis auf einige wenige Ausnahmen erfüllt wurden. Der britische Generalstab stellte im Frühjahr 1922 fest, dass Deutschland de facto entwaffnet war und nicht mehr die militärischen Mittel besaß, einen Krieg in organisierter Weise zu führen. ${ }^{482}$ Unter den britischen Vertretern bei der Interalliierten Militärkontrollkommission (IMKK) in Berlin fanden sich gegenteilige Stimmen. Brigadegeneral Morgan war überzeugt, dass die deutschen Militärs Pläne ausarbeiteten, nach denen die Reichswehr als Nukleus für eine größere Armee dienen sollte, mit der ein Revanchekrieg geführt werden konnte. ${ }^{483}$ Im Foreign Office war man unentschieden, welche Seite der Wahrheit näher kam. ${ }^{484}$ Die französische Regierung wiederum teilte die Befürchtungen, dass Deutschland die militärischen Bestimmungen des Versailler Vertrags gezielt zu unterlaufen versuchte. ${ }^{485}$

Durch die Ruhrbesetzung erhielt die Debatte zusätzliche Nahrung. Die Kontrollkommission musste ihre Tätigkeit vorübergehend einstellen, da die deutsche Regierung nicht mehr kooperierte und die Sicherheit der französischen und belgischen Offiziere gefährdet war. ${ }^{486}$ Daniels vermutete nicht ohne

481 Das Political Intelligence Department des Foreign Office und das Kriegsministerium warnten nach dem Kapp-Putsch und den folgenden Kämpfen zwischen Reichswehr und Roter Armee an der Ruhr vor Anarchie und allgemeiner Unsicherheit, wenn Deutschland seine Truppenstärke wie vorgesehen auf 100000 Mann reduzierte. Das War Office ging davon aus, dass zur Aufrechterhaltung von Ruhe und Ordnung eine Armee von 150000 bis 200000 plus eine Sicherheitspolizei von weiteren bis zu 70000 Mann nötig waren. CARSTEN, Britain and Weimar, S. 44. George Saunders, zu dieser Zeit für die Times in Berlin, schätzte sogar, dass selbst 200000 reguläre Soldaten noch zu wenig waren. Saunders an Steed, 24. Juni 1920. WiLSON (Hrsg.), Saunders on Germany, S. 97.

482 Memorandum des Generalstabs zum Stand der Abrüstung in Deutschland, 31. März 1922, DBFP, I, 20, 202, S. 427.

483 So Morgan in einem Brief an das War Office vom Juni 1922. CARsTEN, Britain and Weimar, S. 53. Morgan bemängelte in diesem Zusammenhang u.a. die lange Dienstzeit von zwölf Jahren, die ihn befürchten ließ, dass die Reichswehr mit einer Struktur aus länger dienenden Freiwilligen ein wirkungsvolles Instrument in der Hand der Reaktionäre werden könnte, da alle höheren Ränge mit Offizieren des Kaiserreichs besetzt waren. Lord Kilmarnock, diplomatischer Vertreter Großbritanniens in Berlin, war dagegen geneigt, Versicherungen der Reichswehrführung zu glauben, dass alle Offiziere die Weimarer Verfassung und die legitime Reichsregierung gegen Angriffe von Rechts und Links verteidigen würden. Ebd., S. 45.

484 Ebd.

485 Die Kontrollkommission stellte immer wieder Verletzungen einzelner Bestimmungen fest. Dabei ging es etwa um die Bestände an Waffen und die Personalstärke der Einwohnerwehren, deren Auflösung die Franzosen verlangten. Diese Forderungen wurden in das Ultimatum der Alliierten vom Mai 1921 an Berlin aufgenommen. Ebd., S. 52 und 54.

486 Es häuften sich Demonstrationen vor den Hotels, in denen Belgier und Franzosen untergebracht waren, sowie Angriffe auf die Offiziere. Williamson, British in Germany, S. 253. 
Grund, dass die Reichswehrführung nun freie Bahn hatte, um so viele Freiwillige auszubilden, wie sie wollte. ${ }^{487}$ Die offenen Aktivitäten illegaler Wehrverbände in Bayern und anderen Teilen des Reiches lenkten das Interesse zusätzlich auf das Thema. ${ }^{488}$ Die französische Regierung veröffentlichte schließlich im September 1923 einen Bericht, in dem zahlreiche Verstöße gegen die Rüstungsauflagen aufgeführt wurden, darunter die Unterhaltung eines Generalstabs, die heimliche Ausbildung junger Männer sowie die Lagerung umfangreicher Waffenbestände, und verlangte ein Einschreiten der Siegermächte. ${ }^{489}$

Nach der Wiederaufnahme ihrer Kontrollen stellte die IMKK am Ende einer Generalinspektion Mitte Dezember 1924 fest, dass Deutschland seinen Entwaffnungsverpflichtungen nicht in vollem Umfang nachkam. ${ }^{490}$ Die alliierten Regierungen verschoben daraufhin die Evakuierung der von den Briten besetzten Kölner Zone, die am 10. Januar 1925 hätte beginnen sollen. ${ }^{491} \mathrm{Im}$ Zuge der zu dieser Zeit beginnenden Sondierungen für einen Sicherheitspakt trat die Entwaffnungsfrage aber auf diplomatischer Ebene in den Hintergrund und wurde schließlich auf der Locarno-Konferenz im Oktober 1925 mit einem Kompromiss gelöst. ${ }^{492}$

Entscheidend für die Darstellung der Problematik in den englischen Zeitungen während dieser Zeit war nicht die Substanz der Vorwürfe. Dass die Deutschen sich auf militärischem Gebiet nicht an alle Punkte des Friedensvertrags hielten und die Alliierten über das wahre Ausmaß ihrer Aktivitäten zu täuschen

487 Daniels an Deakin, 7. Juni 1923, Correspondence between Ralph Deakin and Harold G. Daniels, TNL Archive, TT/FN/1/RD/1.

488 Vgl. z.B. den Bericht von Harold Daniels in The Times, 18. April 1923, „More Irregular Forces".

489 The Daily Mail, 17. September 1923, „German Army - Official French Revelations“; The Times, 19. November 1923, LA „A Day Of Decision“. Die britischen Stellen erfuhren von ihren Offizieren bei der IMKK, die verdächtigen deutschen Einrichtungen inoffizielle Besuche abstatteten, dass heimlich Freiwillige ausgebildet und Reserveoffiziere trainiert wurden. CARSTEN, Britain and Weimar, S. 140f. Es gab aber auch Anzeichen, dass die Abrüstungsfrage in Großbritannien gezielt instrumentalisiert wurde. Die Nachrichtenagentur Renters verbreitete am 29. November 1923 eine Liste mit alarmierenden Details, die am folgenden Tag in allen großen Zeitungen erschien. Von verschiedenen Seiten wurde die Reutersmeldung als offiziell inspiriert angesehen und als ein Versuch der Regierung Baldwin interpretiert, den Vorwurf der Daily Mail im gerade laufenden Wahlkampf zu widerlegen, dass sie eine prodeutsche Politik verfolge. WiLliamson, British in Germany, S. $257 \mathrm{f}$.

490 Darin wurden die von den Franzosen aufgedeckten Verstöße im Wesentlichen bestätigt. Grundmann (Hrsg.), Gebhardt, Bd.4, Teilbd. 1, S.262. Wie Salewski betont, zeichnete der Bericht allerdings ein schiefes Bild, da nur die Verstöße aufgelistet wurden und unerwähnt blieb, dass Deutschland 95 Prozent der Bestimmungen erfüllt hatte. SALEwSKI, Entwaffnung, S. 295.

491 Die Siegermächte konkretisierten und belegten ihre Vorwürfe erst am 4. Juni 1925 in einer weiteren Note an Deutschland.

492 Vgl. ausführlich SALEWSKI, Entwaffnung, S. 315-325. 
versuchten, war unbestritten. Ausschlaggebend war vielmehr das Gewicht, das dem beigemessen wurde. Zentral war dabei die Frage, ob die Verstöße so schwerwiegend waren, dass Deutschland wieder als eine Bedrohung erschien.

Die meisten Redaktionen und ihre Berliner Korrespondenten teilten die Einschätzung des britischen Generalstabs, dass die Reichswehr auf absehbare Zeit nicht fähig war, einen Krieg zu führen, sondern höchstens zur Niederschlagung von Aufständen im Inneren eingesetzt werden konnte. ${ }^{493}$ In einem Memorandum an die Redaktion der Times kam Daniels in Berlin zu dem Schluss, dass den Deutschen das Waffenarsenal für einen Angriffskrieg gegen Frankreich fehlte und Vorbereitungen dafür nicht unbemerkt bleiben würden. ${ }^{494}$ Kritisch beurteilte er das heimliche militärische Training einer großen Zahl Freiwilliger durch die Reichswehr und die allgemein vorherrschende nationalistische Stimmung, die sich auch gegen die Rüstungsauflagen im Versailler Vertrag richtete. ${ }^{495}$ Ausdruck dafür waren die zahlreichen patriotischen und paramilitärischen Verbände wie der „Stahlhelmbund“, die als Reservoir für eine schnelle Aufstockung der Reichswehr dienen konnten und deren Existenz für die Times einen Bruch der Abrüstungsbestimmungen darstellte. ${ }^{496}$ Auch die Erkenntnisse der IMKK, dass noch immer ein Generalstab existierte, wurde als schwerwiegend eingestuft, da dies ein weiterer Hinweis war, dass nach wie vor eine "militärische Rumpforganisation" aufrecht erhalten wurde, die innerhalb kurzer Zeit ausgedehnt werden konnte. ${ }^{497}$ Normale, freundschaftliche Beziehungen zwischen Deutschland und den Alliierten seien aber nur möglich, wenn die Deutschen offen mit ihren weiter hartnäckig vorhandenen militärischen Traditionen brechen würden, betonte die Times. ${ }^{498}$

$\mathrm{Zu}$ einem weniger ambivalenten Ergebnis kam Voigt im Manchester Guardian. ${ }^{499}$ Der Einfluss des deutschen Generalstabs sei schon zu Zeiten des

493 The Daily Telegraph, 3. Januar 1923; The Daily Herald, 30. November 1923, „Germany’s Arms“; THE TiMes, 4. April 1924, LA „German Disarmament“; THE MANCHESTER GuARDiAn, 7.Januar 1925, „German Disarmament - The Points Of Allied Complaint Examined“.

494 „Memorandum by Harold Daniels to Harold Williams re German Disarmament“, undatiert [nach 1923], TNL Archive, BNS/3.

495 Ebd.

496 The Times, 4. April 1924, LA „German Disarmament“. Sie verlangte deshalb eine Fortsetzung der alliierten Kontrollen, was ihr eine Beschwerde vom Presseattaché der deutschen Botschaft, Dufour-Feronces, einbrachte, da die Reichsregierung zu diesem Zeitpunkt auf ein Ende der militärischen Inspektionen drängte. Der Außenpolitikchef Williams bemerkte dazu, es sei „rather intolerable“, dass die Deutschen glaubten, die Times stehe in jeder strittigen Frage an ihrer Seite. Williams an Daniels, 9. April 1924, Williams Papers, Correspondence with Harold G. Daniels, TNL Archive, HW/1.

497 Englisch „skeleton military organization“.

498 The Times, 8. Januar 1925, LA „German Disarmament“.

499 The MANChester Guardian, 7.Januar 1925, „German Disarmament - The Points Of Allied Complaint Examined“ und 8. Juni 1925, „Scope Of Disarmament Demands - A Burden Of Numerous Trivialities“. 
Kaiserreichs überschätzt worden. Das Gegenstück, die Heeresleitung unter dem Oberkommandierenden der Reichswehr, General von Seeckt, trete zudem nur bei Manövern oder in Krisenzeiten zusammen. Die Ausbildung von Freiwilligen habe seit dem Ende des Ruhrkampfes rapide abgenommen und die militaristischen Organisationen würden nach und nach von allein verschwinden. Der Note an Deutschland, in der die alliierten Regierungen im Juni 1925 bekräftigten, dass die Verstöße gegen die Entwaffnungsbestimmungen eine Gefahr für den Frieden darstellten, widersprach der $M G$ vehement: „there is not the faintest indication that Germany really has the beginnings of an army capable of defence against even a second-class Power. The notion that she is herself capable of attacking [...] is simply fantastic. "500 Der Daily Herald äußerte indirekt sogar Verständnis für die verdeckten militärischen Aktivitäten Berlins. Seit dem Abschluss des Versailler Vertrags werde Deutschland zur Entwaffnung gezwungen, während die benachbarten Mächte weiter für einen Krieg rüsteten. ${ }^{501}$

Eine Ausnahme bildete auch in diesem Fall die Daily Mail. Das Massenblatt Rothermeres baute auf den Enthüllungen eine regelrechte Kampagne nach dem bereits mehrfach erprobten Muster auf, in der den Deutschen wiederum die Rolle des „reuelosen Sünders“ zugewiesen und mit der Verallgemeinerung gearbeitet wurde, die gesamte Bevölkerung unterstütze die Militärs. ${ }^{502}$ In regelmäßigen Abständen erschienen seit dem französischen Bericht vom September 1923 Artikel über immer neue Verstöße gegen die Rüstungsauflagen, die den Eindruck verstärkten, dass Deutschland auf Revanche sann, deshalb heimlich rüstete und die Gefahr akut war. ${ }^{503}$ Percival Phillips sprach in einer Serie von Reportagen davon, dass eine Verschwörung gegen die Alliierten im Gang sei, an dessen Spitze die „Militärclique“ stehe, die seit dem Krieg „nichts vergessen und nichts vergeben" habe. ${ }^{504}$ Die Mail berief sich dabei auch auf die Warnungen des britischen Generals Morgan und verlangte, dass die Siegermächte vorerst das Rheinland und die Brückenköpfe besetzt halten müssten. Andernfalls sei der Frieden nicht länger als ein Jahr sicher. ${ }^{505}$

500 Ebd., 6. Juni 1925, LA „German Disarmament“.

501 The Daily Herald, 6. Juni 1925, LA „From The Workers' Point Of View - A Tangled Web“.

502 The Daily Mail, 4. März 1925, LA „Germany Still Arming - The Problem For The Allies".

503 Ebd., 20. September 1923, „German Army Hidden Munitions“ und 12. November 1923, „Berlin Flouts The Allies - Secret Arming To Continue“ und 19. November 1923, „Germany’s Fresh Defiance“ und 7. April 1924, „The Secret Army - Machine-Gun Practice For, Volunteers“".

504 So Phillips zum Auftakt in der Daily Mail am 2. Januar 1925, „German Arms Plots - Trick That Do Not Deceive Allied Officers“. Die weiteren Teile erschienen am 7.Januar 1925, "Gun-Making Machines - Krupp’s Excuses To The US“ und 13. Januar, „New German Armies - Trained By Secret Societies“.

505 Ebd., 4. März 1925, LA „Germany Still Arming - The Problem For The Allies“. 
Diese Warnungen waren nicht aus der Luft gegriffen. Die illegale Rüstung war gedeckt durch ein breites Sympathisantenfeld in der deutschen Politik, Industrie, Bürokratie, Justiz und Presse. ${ }^{506}$ Heimlich arbeiteten Militärs und Rüstungsfirmen an der Entwicklung verbotener Waffen wie Panzer, Flugzeuge und U-Boote. ${ }^{507}$ Außerdem existierte eine so genannte „Schwarze Reichswehr", eine geheime Ersatzarmee, die aus Einwohnerwehren, Freikorps und nicht aufgelösten Verbänden der kaiserlichen Armee bestand und von der Wirtschaft oder von Privatleuten finanziert wurde.508 Ohne mehrjährige Vorbereitung bestand jedoch keine Aussicht, dass die Reichswehr in einem militärischen Konflikt gegen die Westmächte bestehen konnte. Wegen der deutschen Unterlegenheit war sich die britische Botschaft in Berlin sicher, dass, trotz der bellizistischen Rhetorik der nationalistischen Parteien auf der Rechten und obwohl weite Teile der Öffentlichkeit eine Aufrüstung gerade nach der Ruhrbesetzung befürworteten, niemand ernsthaft daran dachte, erneut einen Krieg zu beginnen. 509

Die englischen Pressestimmen in der Abrüstungsdebatte spiegeln die allgemein herrschende Meinung in Großbritannien wider, nach der Deutschland nicht mehr als militärische Bedrohung empfunden wurde. ${ }^{510}$ Sie zeigen darüber hinaus, wie groß die Bereitschaft inzwischen war, zu Gunsten verbesserter Beziehungen über Verletzungen des Versailler Vertrags hinwegzusehen. Zugleich blieb aber der Eindruck, dass aufeinander folgende Reichsregierungen nicht mit offenen Karten spielten und der Militarismus in Deutschland weiterhin eine starke Kraft war. Letzteres erschwerte die Überwindung des gegenüber der deutschen Außenpolitik nach wie vor bestehenden Misstrauens, so das Fazit der Times. „These delinquincies strengthen the natural fears, suspicions, and anxieties that still remain.“511

506 KÜHNL, Weimarer Republik, S. 61.

507 Ebd., S. 63.

508 Ebd., S. 64. Hinzu kam die Sicherheitspolizei, die dem Innenministerium unterstand, sich aber aus ehemaligen Offizieren und Unteroffizieren rekrutierte. Außerdem existierte im Reichswehrministerium eine geheime Abteilung, die dafür Sorge tragen sollte, dass genug Ausrüstung für ein Heer von einer Million Mann vorgehalten wurde. KLEINE-AHLBRANDT, Burden, S. 79. Vgl. zu diesem Problemkomplex neuerdings umfassend NAKatA, Grenz- und Landesschutz.

509 Knox, Berlin, an Lampson, 8. Mai 1924, PRO, FO 371/9825, C6157/2977/18.

510 „British opinion generally holds that, whatever the degree of Germany's will to peace, or lack of it, she is materially powerless to attack any of her neighbours, and will remain so for years to come." So fasste der Diplomatic Correspondent des Telegraph, Maurice Gerothwohl, die Stimmung zusammen. The Daily Telegraph, 8. Juni 1925.

511 The Times, 6. Juni 1925, LA „The Evacuation Of Cologne“. 